\title{
Precipitation in aqueous mixtures with addition of strongly hydrophilic or hydrophobic solute
}

\author{
Ryuichi Okamoto and Akira Onuki \\ Department of Physics, Kyoto University, Kyoto 606-8502, Japan
}

(Dated: February 26, 2018)

\begin{abstract}
We examine phase separation in aqueous mixtures due to preferential solvation with a low-density solute (hydrophilic ions or hydrophobic particles). For hydrophilic ions, preferential solvation can stabilize water domains enriched with ions. This precipitation occurs in wide ranges of the temperature and the average composition above a critical solute density $n_{\mathrm{p}}$, where the mixture solvent would be in a one-phase state without solute. The volume fraction of precipitated domains tends to zero as the average solute density $\bar{n}$ is decreased to $n_{\mathrm{p}}$ or as the interaction parameter $\chi$ is decreased to a critical value $\chi_{\mathrm{p}}$. If we start with one-phase states with $\bar{n}>n_{\mathrm{p}}$ or $\chi>\chi_{\mathrm{p}}$, precipitation proceeds via homogeneous nucleation or via heterogeneous nucleation, for example, around suspended colloids. In the latter case, colliod particles are wrapped by thick wetting layers. We also predict a first-order prewetting transition for $\bar{n}$ or $\chi$ slightly below $n_{\mathrm{p}}$ or $\chi_{\mathrm{p}}$.

PACS numbers: 82.45.Gj, 61.20.Qg, 64.75.Cd, 81.16.Dn
\end{abstract}

\section{INTRODUCTION}

In fluid mixtures composed of water and a less polar organic liquid, phase separation behavior can be drastically changed by a small amount of salt $[1]$ addition of a $10^{-3}$ mole fraction of hydrophilic salt like $\mathrm{NaCl}$, the coexistence curve is typically shifted by a few Kelvins upward in the USCT case or downward in the LSCT case. In some aqueous mixtures, even if they are miscible at all $T$ at atmosphere pressure without salt, addition of a small amount of salt gives rise to reentrant phase separation behavior $[3-6]$.

With ions added, moreover, many groups have also found long-lived heterogeneities (sometimes extending over a few micrometers) in one-phase states [9] and a third phase visible as a thin solid-like plate at a liquidliquid interface in two-phase states [10]. A representative system is a mixture of $\mathrm{H}_{2} \mathrm{O}$ (or $\mathrm{D}_{2} \mathrm{O}$ ), tri-methylpyridine (3MP), and $\mathrm{NaBr}$. The heterogeneities have been detected by dynamic light scattering [11], indicating spontaneous formation of aggregates or domains with small diffusion constants. Their size and the volume fraction are $50 \AA$ and $10^{-6}$ as a typical example. The third phase have been observed also in isobutylic acid (IBA)-water mixtures without added salt, where the isobutyric acid partly dissociates into Butyrate ${ }^{-}$and $\mathrm{H}^{+}$ions. In addition, a drastic decrease in the ion mobility was observed in IBA-water for high contents of IBA 12]. Recently, mesophases have been observed when sodium tetraphenylborate $\mathrm{NaBPh}_{4}$ was added to $\mathrm{D}_{2} \mathrm{O}-3 \mathrm{MP}$ at about $0.1 \mathrm{M}$ [13]. This salt dissociates into hydrophilic $\mathrm{Na}^{+}$and hydrophobic $\mathrm{BPh}_{4}^{-}$. The latter ion consists of four phenyl rings bonded to an ionized boron.

Dramatic ion effects are ubiquitous in various soft materials such as polymers, gels, colloids, and mixtures containing ionic surfactants. For example, complex effects are known to be induced in polyelectrolytes when a second fluid component (cosolvent) is added to water. In particular, precipitation of DNA has been widely ob- served with addition of alcohol such as ethanol to water 14. Here the alcohol added is excluded from condensed DNA, which suggests solvation-induced wetting of DNA by water. We also mention observations of crystal formation of micron-sized water-rich droplets in a less polar oil-rich phase with addition of $\mathrm{HBr}$ [15]. The mechanism of this ordering was ascribed to the Coulomb interaction due to asymmetric partitioning of cations and anions in water and oil [16].

In these phenomena, the solvation (or hydration) interaction among ions and polar molecules should play a major role. Small hydrophilic ions are solvated by several water molecules [17 -19] and the resultant solvation chemical potential per ion much exceeds the thermal energy $k_{B} T$ and strongly depends on the ambient composition of the solvent. On the contrary, some hydrophobic particles dislike to be in contact with water molecules, which tend to form aggregates in water and are more soluble in oil than in water. In aqueous mixtures, a hydrophobic ion should be surrounded by oil molecules, which explains the mesophase formation observed by Sadakane et al [13]. Hydrophilic and hydrophobic particles strongly affect the surrounding hydrogen bonding network in different manners [17] and the solvation is highly cooperative. Furthermore, when a small amount of water was added to methanol-cyclohexane, the coexistence curve was largely shifted [20] and a water-induced, methanol-rich wetting layer appeared (which was nonexistent without water) 21]. These findings indicate strongly selective molecular interactions between water and the two components.

Obviously, the preferential solvation should strongly affect the phase transition behavior in aqueous mixtures with a small amount of hydrophilic or hydrophobic solute, though this aspect has not been well studied. Recently, some efforts have been made to elucidate the solvation effects in phase separation in mixture solvents in electrolytes 22 28], polyelectrolytes 29], and ionic surfactants 30]. A review on the static properties was presented [31]. In the dynamics, a number of problems still 
remain unexplored [27, 28].

In this paper, we present a theory of solvation-induced phase separation with hydrophilic or hydrophobic solute. Thus we aim to explain the observed heterogeneities in aqueous mixtures [9]. We may suppose hydrophilic monovalent ion pairs such as $\mathrm{Na}^{+}$and $\mathrm{Cl}^{-}$in a binary mixture of water and a less polar component (called oil). As is well-known, hydrophilic ions induce clustering of water molecules around them on microscopic scales, forming a solvation shell [17-19]. We shall see that strongly hydrophilic ions can moreover induce formation of water-rich domains on macroscopic scales for sufficiently strong preference of water over oil. This can occur even when the mixture is outside the coexistence curve without ions.

The organization of this paper is as follows. In Sec.II, two-phase coexistence induced by the preferential solvation will be studied numerically and theoretically, where the electrostatic interaction does not appear explicitly. In Sec.III, inhomogeneous profiles such as interfaces will be calculated. We will show that a precipitated droplet can be stable only above a minimum radius due to the surface tension effect and that a solvation-induced prewetting transition occurs far from the coexistence curve without solute. In Sec.IV, two-phase coexistence with hydrophilic ions will be examined in the presence of the charge effect. In Sec.V, we will investigate the precipitation from onephase states taking place as homogeneous nucleation. In Sec.IV, we will investigate adsorption and precipitation on colloid surfaces.

\section{PHASE SEPARATION WITH STRONGLY SELECTIVE SOLUTE}

Neglecting electrostatic interaction but accounting for the solvation interaction, we first consider a binary mixture composed of water and a less polar component in a cell with a fixed volume $V$. The second component will be simply called oil hereafter. Ions will be treated as a dilute third component (solute) with density $n(\boldsymbol{r})$. We may also suppose strongly hydrophilic or hydrophobic neutral particles (possibly with a complex structure) added in an aqueous binary mixture. Such particles can be solvated by a certain number of water or oil molecules depending on whether they are hydrophilic or hydrophobic.

In the following, we will suppose hydrophilic ions or particles. However, our results can be equally applicable to hydrophobic ions or particles if water and oil are exchanged (or $\phi$ is replaced by $1-\phi$ ). The Boltzmann constant $k_{B}$ will be set equal to unity.

\section{A. Conditions of two phase coexistence with solute}

The volume fractions of water, oil, and solute are written as $\phi(\boldsymbol{r})$ and $\phi^{\prime}(\boldsymbol{r})$, and $v_{I} n(\boldsymbol{r})$, respectively, where $v_{I}$ is the solute volume. If the two solvent species have the same molecular volume $v_{0}$, their densities are $\phi / v_{0}$ and $\phi^{\prime} / v_{0}$. The solvent diameter is of order $a=v_{0}^{1 / 3}$. The space-filling condition is written as

$$
\phi+\phi^{\prime}+v_{I} n=1
$$

The solute volume fraction is assumed to be small or $v_{I} n \ll 1$. This is more easily satisfied if the solute size is smaller than that of solvent or $v_{I}<v_{0}$. In this paper, to simplify the calculations, we thus set

$$
\phi^{\prime}=1-\phi .
$$

The free energy density $f_{\text {tot }}(\phi, n)$ for the composition $\phi$ and the solute density $n$ consists of three parts as

$$
f_{\text {tot }}(\phi, n)=f(\phi)+T n \ln \left(n \lambda^{3}\right)-T g n \phi .
$$

The first term is assumed to be of the Bragg-Williams form for a fluid mixture [32],

$$
f(\phi)=\frac{T}{v_{0}}[\phi \ln \phi+(1-\phi) \ln (1-\phi)+\chi \phi(1-\phi)],
$$

where $\chi$ is the interaction parameter dependent on $T$. The length $a=v_{0}^{1 / 3}$ represents the molecular diameter

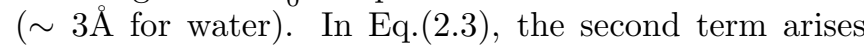
from the solute translational entropy. The $\lambda$ is the thermal de Broglie length (but the terms linear in $n$ are irrelevant and $\lambda^{3}$ may be replaced by $v_{0}$ in the following). The third term arises from the solute preference of water over oil. The parameter $g$ is assumed to much exceed unity $(\gg 1)[23$. We fix the average densities of the constituent components, which are expressed as

$$
\bar{\phi}=\int d \boldsymbol{r} \phi / V, \quad \bar{n}=\int d \boldsymbol{r} n / V .
$$

In our theory, $\chi, \bar{\phi}$, and $\bar{n}$ are relevant control parameters. Changing $\chi$ through the critical value $\chi_{c}=2$ is equivalent to changing $T$ through the solvent critical temperature $T_{c}$.

In two phase coexistence in equilibrium, let the composition and the solute density be $\left(\phi_{\alpha}, n_{\alpha}\right)$ and $\left(\phi_{\beta}, n_{\beta}\right)$ in phases $\alpha$ and $\beta$ with $\phi_{\beta}<\bar{\phi}<\phi_{\alpha}$ and $n_{\beta}<\bar{n}<n_{\alpha}$. Here we give simple thermodynamic arguments. We define the chemical potentials as $h=\partial f_{\text {tot }} / \partial \phi$ and $\mu=\partial f_{\text {tot }} / \partial n$. From Eq.(2.3) we obtain

$$
\begin{aligned}
h & =f^{\prime}(\phi)-T g n, \\
\mu & =T\left[\ln \left(n \lambda^{3}\right)+1-g \phi\right],
\end{aligned}
$$

where $f^{\prime}=\partial f / \phi$. First, the homogeneity relation $\mu\left(\phi_{\alpha}, n_{\alpha}\right)=\mu\left(\phi_{\beta}, n_{\beta}\right)$ for solute gives rise to

$$
n_{\alpha}=A_{0} e^{g \phi_{\alpha}}, \quad n_{\beta}=A_{0} e^{g \phi_{\beta}} .
$$

The coefficient $A_{0}=\lambda^{-3} \exp (\mu / T-1)$ is determined from the conservation of the solute number in Eq.(2.5) as

$$
A_{0}=\bar{n} /\left\langle e^{g \phi}\right\rangle
$$


where $\langle\cdots\rangle$ denotes the space average in the cell. Let $\gamma_{\alpha}$ be the volume fraction of phase $\alpha$. Neglecting the volume of the interface region, we obtain

$$
\left\langle e^{g \phi}\right\rangle=\gamma_{\alpha} e^{g \phi_{\alpha}}+\left(1-\gamma_{\alpha}\right) e^{g \phi_{\beta}}
$$

From Eq.(2.5) $\gamma_{\alpha}$ is expressed in terms of $\bar{\phi}$ and $\bar{n}$ as

$$
\begin{aligned}
\gamma_{\alpha} & =\left(\bar{\phi}-\phi_{\beta}\right) / \Delta \phi \\
& =\left(\bar{n}-n_{\beta}\right) / \Delta n .
\end{aligned}
$$

The differences between the two phases are written as

$$
\Delta \phi=\phi_{\alpha}-\phi_{\beta}>0, \quad \Delta n=n_{\alpha}-n_{\beta}>0 .
$$

With the aid of Eq.(2.8), the ratio $n_{\alpha} / \bar{n}$ is written in terms of the compositions as

$$
n_{\alpha} / \bar{n}=\Delta \phi /\left[\left(\bar{\phi}-\phi_{\beta}\right)+\left(\phi_{\alpha}-\bar{\phi}\right) e^{-g \Delta \phi}\right] .
$$

For not small $\Delta \phi$, we find $n_{\alpha} / n_{\beta}=e^{g \Delta \phi} \gg 1$ and $\Delta n \cong$ $n_{\alpha}$. Namely, the solute density is much higher in phase $\alpha$ than in phase $\beta$. The solvation part $-T g n_{\alpha} \phi$ of the free energy density is significant in phase $\alpha$ even for small $\bar{n}$.

Next, the homogeneity relation $h\left(\phi_{\alpha}, n_{\alpha}\right)=h\left(\phi_{\beta}, n_{\beta}\right)$ for the solvent composition is written as

$$
\begin{aligned}
h & =f^{\prime}\left(\phi_{\alpha}\right)-T g n_{\alpha} \\
& =f^{\prime}\left(\phi_{\beta}\right)-T g n_{\beta} .
\end{aligned}
$$

In equilibrium, we also require minimization of the grand potential density $\omega$ defined by

$$
\begin{aligned}
\omega & =f_{\text {tot }}-h \phi-\mu n \\
& =f-h \phi-T n,
\end{aligned}
$$

where the second line follows from Eq.(2.7). In the two phases, $\omega$ assumes the same value so that

$$
f\left(\phi_{\alpha}\right)-f\left(\phi_{\beta}\right)-T \Delta n=h \Delta \phi .
$$

We may derive Eqs.(2.15) and (2.17) from minimization of the total free energy $F$ under Eq.(2.5). Using $\gamma_{\alpha}$ we express $F$ as

$$
F / V=\gamma_{\alpha} f_{\text {tot }}\left(\phi_{\alpha}, n_{\alpha}\right)+\left(1-\gamma_{\alpha}\right) f_{\text {tot }}\left(\phi_{\beta}, n_{\beta}\right),
$$

where the surface free energy is neglected. If use is made of the relation $n\left(\ln \left(n \lambda^{3}\right)-g \phi\right)=n \ln A_{0}$ in the two phases, the above expression is rewritten as

$$
\begin{aligned}
F / V & =\gamma_{\alpha} f\left(\phi_{\alpha}\right)+\left(1-\gamma_{\alpha}\right) f\left(\phi_{\beta}\right)+T \bar{n} \ln \left(\bar{n} \lambda^{3}\right) \\
& -T \bar{n} \ln \left[\gamma_{\alpha} e^{g \phi_{\alpha}}+\left(1-\gamma_{\alpha}\right) e^{g \phi_{\beta}}\right] .
\end{aligned}
$$

The third term in the first line is a constant at constant $\bar{n}=\langle n\rangle$ and is irrelevant, but the fourth term in the second line is a singular solvation contribution at fixed $\bar{n}=\langle n\rangle$ relevant for large $g$ (even for small $\bar{n}$ ). Since $\bar{\phi}=\langle\phi\rangle$ is also fixed, we should minimize

$$
\tilde{\omega}=F / V-h\left[\gamma_{\alpha} \phi_{\alpha}+\left(1-\gamma_{\alpha}\right) \phi_{\beta}-\bar{\phi}\right]
$$
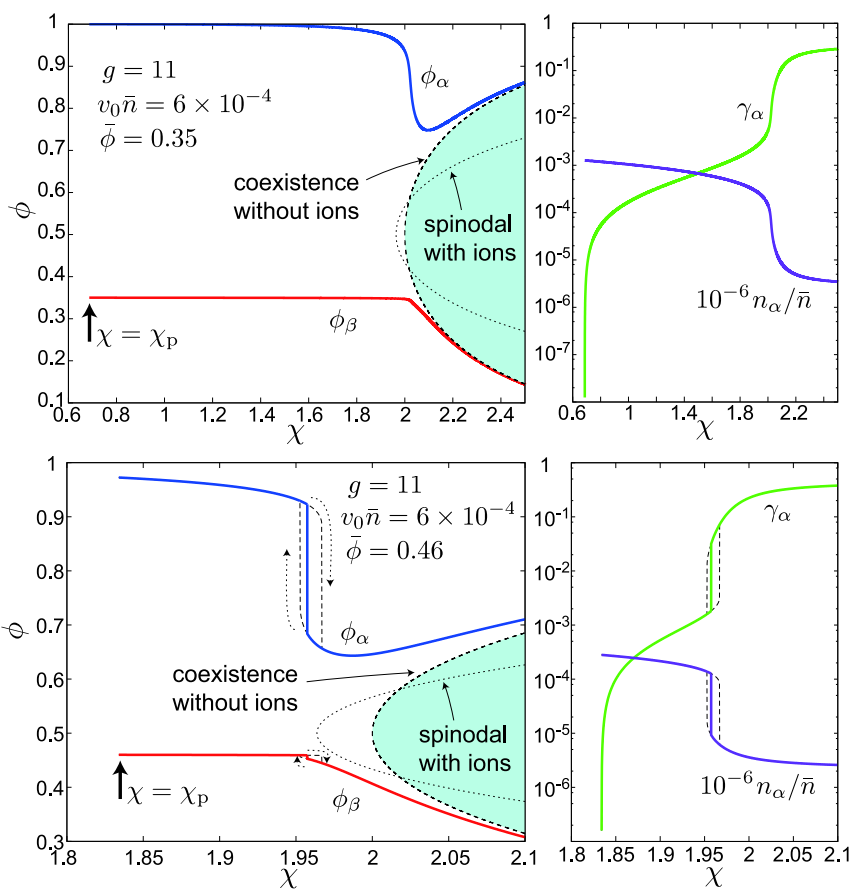

FIG. 1: (Color online) Left:Compositions $\phi_{\alpha}$ and $\phi_{\beta}$ vs $\chi$. Right:Semi-logarithmic plots of volume fraction $\gamma_{\alpha}$ and normalized ion density $n_{\alpha} / \bar{n}$ of the water-rich phase $\alpha$ vs $\chi$. Here $\bar{n}=6 \times 10^{-4} v_{0}^{-1}$ and $g=11$. Precipitation occurs for $\chi_{\mathrm{p}}<\chi \lesssim 2$. For $\bar{\phi}=0.35$ (top), $\phi_{\alpha}$ changes continuously. For $\bar{\phi}=0.46$ (bottom), $\phi_{\alpha}$ jumps at $\chi \cong 2$. Plotted in the left also are the coexisting region without solute (in blue) and the spinodal curve with solute (see Eqs.(3.7) and (5.2)) [23].

with respect to $\phi_{\alpha}, \phi_{\beta}$, and $\gamma_{\alpha}$. Here $h$ appears as the Lagrange multiplier. With the aid of the expression (2.19), the minimum conditions, $\partial \tilde{\omega} / \partial \phi_{\alpha}=\partial \tilde{\omega} / \partial \phi_{\beta}=$ $\partial \tilde{\omega} / \partial \gamma_{\alpha}=0$, readily lead to Eqs.(2.15) and (2.17).

Without solute $\bar{n}=0$, two-phase coexistence is possible only for $\chi>2$. Thus, in the appendix A, we will perform the Taylor expansions of $\phi_{\alpha}$ and $\phi_{\beta}$ with respect to $\bar{n}$ for $\chi>2$. We shall see that the water-rich coexistence branch is much deformed even by a very small amount of highly preferential solute (as can be seen in Fig.1 below).

\section{B. Numerical results of precipitation}

Next we give numerical results on the phase behavior of our system. We will set $g=11$ mostly, but will also present additional results for $g=15$ in Fig. 3 below. The solute density $n$ will be measured in units of $v_{0}^{-1}$.

In Fig.1, we display $\phi_{\alpha}$ and $\phi_{\beta}$ in the left panels and $n_{\alpha}$ and $\gamma_{\alpha}$ in the right panels as functions of $\chi$ (or $T$ experimentally) at $\bar{n}=6 \times 10^{-4} v_{0}^{-1}$. Due to the nonlinear solute effect, a precipitation branch appears in the range,

$$
\chi_{\mathrm{p}}(\bar{\phi}, \bar{n})<\chi<2,
$$



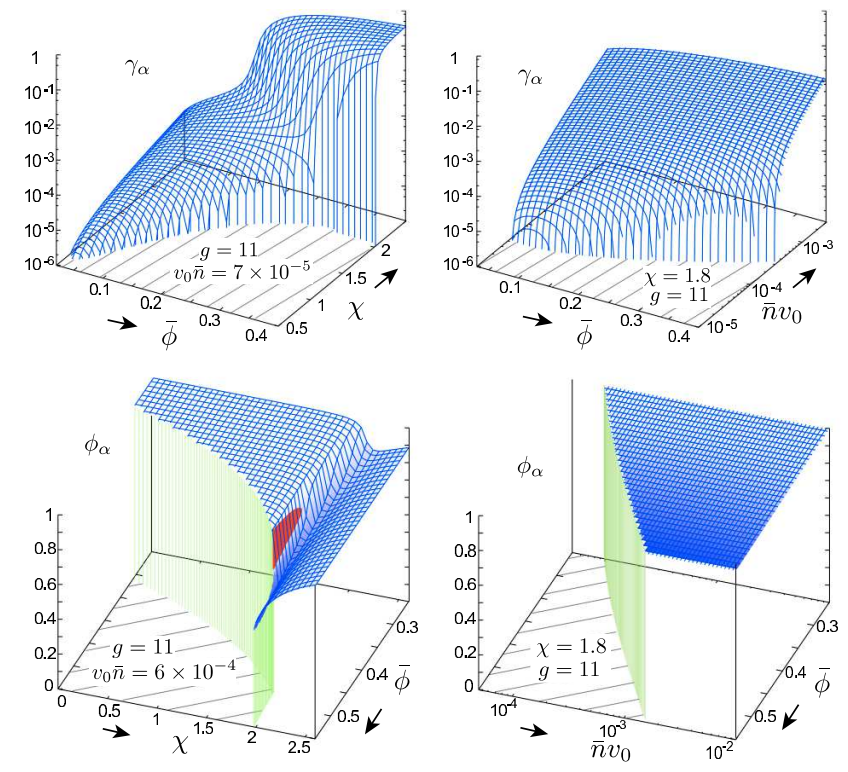

FIG. 2: (Color online) Volume fraction $\gamma_{\alpha}$ (top) and composition $\phi_{\alpha}$ (bottom) of the water-rich phase $\alpha$ in the $\chi-\bar{\phi}$ plane at fixed $\bar{n}$ (left) and in the $\bar{n}-\bar{\phi}$ plane at fixed $\chi$ (right), where $g=11$. Precipitation occurs for $\chi>\chi_{\mathrm{p}}(\bar{\phi}, \bar{n})$ (left) and $\bar{n}>n_{\mathrm{p}}(\bar{\phi}, \chi)$ (right) outside the slashed regions. In the left bottom, a first-order transition occurs for $\chi \cong 1.95$ and $\bar{\phi}>0.395$ (in red). See the bottom plates in Fig.1.

where the solvent would be in one-phase states without solute. Here $\gamma_{\alpha}$ tends to zero as $\chi \rightarrow \chi_{\mathrm{p}}$, where the lower bound $\chi_{\mathrm{p}}$ depends on $\bar{\phi}$ and $\bar{n}$. This branch appears under the condition $e^{g(1-\bar{\phi})} \gg 1$ (see the next subsection). For large $g(=11$ here $)$, the precipitated domains become solute-rich (salted water for hydrophilic ions) with

$$
\phi_{\alpha} \cong 1, \quad n_{\alpha} \gg n_{\beta}=e^{-g \Delta \phi} n_{\alpha} .
$$

The right panels are on semi-logarithmic scales. With decreasing $\chi$, we can see an increase in the solute density $n_{\alpha}$ and an decrease in the volume fraction $\gamma_{\alpha}$. Details of the figure are as follows. (i) For $\bar{\phi}=0.35$ (top), $\phi_{\alpha}$ changes rather abruptly around $\chi \sim 2$ but continuously as a function of $\chi$, being minimum at $\chi=2.05$. The $n_{\alpha}$ increases up to $0.381 v_{0}^{-1}$ as $\chi \rightarrow \chi_{\mathrm{p}}=0.687$. (ii) For $\bar{\phi}=0.46$ (bottom), the precipitation branch much shrinks with $\chi_{\mathrm{p}}=1.834$, while $n_{\alpha}=0.0842 v_{0}^{-1}$ at $\chi=$ $\chi_{\mathrm{p}}$. For this larger $\bar{\phi}, \phi_{\alpha}$ changes discontinuously on a hysteresis loop in the range $1.953<\chi<1.967$. If $F$ in Eq.(2.18) is minimized, a first-order transition is found to occur at $\chi=1.957$. Even for $\chi>2$, the two-phase coexistence is much deformed by the solute.

In Fig. 2 , we show $\gamma_{\alpha}$ and $\phi_{\alpha_{-}}$in the $\chi-\bar{\phi}$ plane at $\bar{n}=$ $6 \times 10^{-4} v_{0}^{-1}$ (left) and in the $\bar{n}-\bar{\phi}$ plane at $\chi=1.8$ (right). For fixed $\chi$ in the $\bar{n}-\bar{\phi}$ plane, precipitation occurs for

$$
\bar{n}>n_{\mathrm{p}}(\bar{\phi}, \chi)
$$

where $\gamma_{\alpha}$ tends to zero as $\bar{n} \rightarrow n_{\mathrm{p}}$. This minimum solute density $n_{\mathrm{p}}$ depends on $\bar{\phi}$ and $\chi$. As the crossover to the
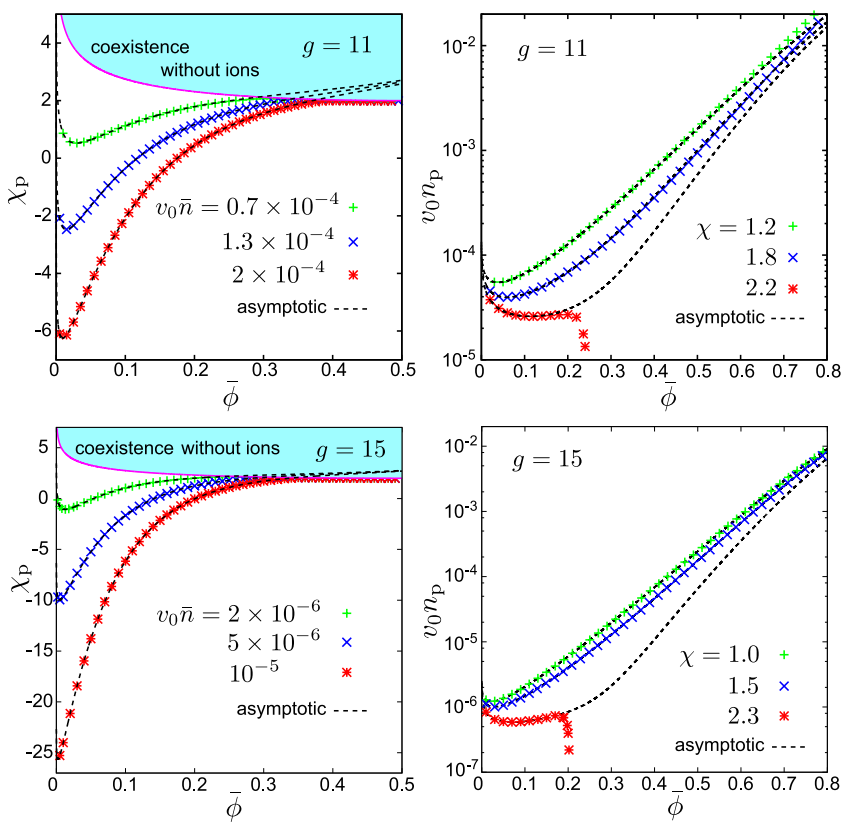

FIG. 3: (Color online) Left: $\chi_{\mathrm{p}}(\bar{\phi}, \bar{n})$ vs $\bar{\phi}$ for three values of $\bar{n}$ at $g=11$ (top) and 15 (bottom), which nearly coincide with the asymptotic formula (2.33) (dotted line) for $\bar{\phi}<0.35$ and converge to the coexistence curve for larger $\bar{\phi}$. Coexistence region without ions is also shown (in blue). Right: $v_{0} n_{\mathrm{p}}(\bar{\phi}, \chi)$ vs $\bar{\phi}$ on a semi-logarithmic scale for three values of $\chi$ at $g=$ 11 (top) and 15 (bottom), It coincides with the asymptotic formula (2.34) (dotted line) for $\chi<2$ and tends to zero at the coexistence composition, 0.249 (top) or 0.204 (bottom).

asymptotic behavior in Eq.(2.22), $\phi_{\alpha}$ becomes appreciably smaller than 1 for $\chi \sim 2$ or for $\bar{\phi} \sim 0.5$. For large $\bar{\phi} \gtrsim 0.5$, the precipitation branch shrinks to vanish for small solute densities, where water molecules are already abundant around solute particles in one-phase states.

In Fig.3, at $g=11$ and 15 , we show $\chi_{\mathrm{p}}(\bar{\phi}, \bar{n})$ vs $\bar{\phi}$ for three values of $\bar{n}$ (left) and $n_{\mathrm{p}}(\bar{\phi}, \chi)$ vs $\bar{\phi}$ for three values of $\chi$ (right). These quantities decrease dramatically at small $\bar{\phi}$ and their magnitudes strongly depend on $g$. Even for $\chi>2, n_{\mathrm{p}}$ can be calculated outside the water-rich branch of the coexistence curve and it tends to zero as $\bar{\phi}$ approaches the coexistence composition without solute. Theoretical explanations of their behavior will be given in the following subsections.

\section{Theory of asymptotic behavior}

We present a theory on the asymptotic behavior of the precipitation branch for $g \gg 1$ in the region $\chi<2$. At its starting point, we assume the branch satisfying Eq.(2.22) and confirm its existence self-consistently. Since $\phi_{\alpha} \cong 1$, the logarithmic term $(\propto(1-\phi) \ln (1-\phi))$ in the free energy density (2.4) is crucial in phase $\alpha$.

We first neglect the term $-T g n_{\beta}$ in Eq.(2.15) from $g v_{0} n_{\beta} \ll 1$ and the term $f\left(\phi_{\alpha}\right)$ in Eq.(2.17) from $f\left(\phi_{\alpha}\right) \sim$ 


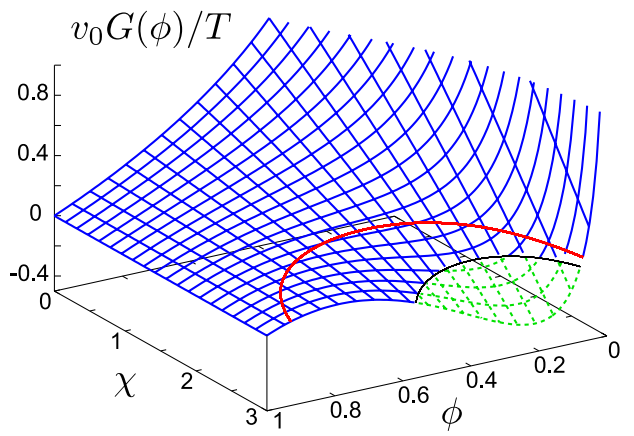

FIG. 4: (Color online) $v_{0} G(\phi) / T$ defined in Eq.(2.26) in the $\phi-\chi$ plane. It is negative only in the inner green region, where the minimum of $\chi$ is 2.455 at $\phi=0.285$. It is positive outside it in the blue region. The coexistence curve without solute is written in red, on which the minimum of $\chi$ is 2 at $\phi=0.5$.

$T\left(1-\phi_{\alpha}\right) \ln \left(1-\phi_{\alpha}\right)$. Note that $g v_{0} n_{\beta} \sim 10^{-2}$ in Fig.1. It follows a simplified equation,

$$
h \cong f^{\prime}\left(\phi_{\beta}\right) \cong-\left[f\left(\phi_{\beta}\right)+T n_{\alpha}\right] /\left(1-\phi_{\beta}\right) .
$$

This determines the solute density $n_{\alpha}$ in phase $\alpha$ as a function of $\phi_{\beta}$ in the form,

$$
n_{\alpha} \cong G\left(\phi_{\beta}\right) / T,
$$

where $G(\phi)$ is defined by

$$
\begin{aligned}
G(\phi) & =-f(\phi)-(1-\phi) f^{\prime}(\phi) \\
& =-\left(T / v_{0}\right)\left[\ln \phi+\chi(1-\phi)^{2}\right] .
\end{aligned}
$$

Using the free energy (2.4), we obtain the second line and plot it in the $\phi$ - $\chi$ plane in Fig.4. It is positive outside the coexistence curve, ensuring $n_{\alpha}>0$ in Eq.(2.25).

In Eq.(2.15), we next use Eq.(2.4) for $f^{\prime}\left(\phi_{\alpha}\right)$ to obtain

$$
h \cong v_{0}^{-1} T\left[-\ln \left(1-\phi_{\alpha}\right)-\chi\right]-T g n_{\alpha} \cong f^{\prime}\left(\phi_{\beta}\right),
$$

where the logarithmic term $(\propto \ln (1-\phi))$ balances with the solvation term $\left(\propto g n_{\alpha}\right)$. Use of Eq.(2.26) gives

$$
1-\phi_{\alpha} \cong A_{\beta} \exp \left[-g v_{0} G\left(\phi_{\beta}\right) / T\right]
$$

where the coefficient $A_{\beta}$ is given by

$$
A_{\beta}=\exp \left[-\chi-v_{0} f^{\prime}\left(\phi_{\beta}\right) / T\right],
$$

so $A_{\beta}$ is of order unity. The factor $\exp \left[-g v_{0} G\left(\phi_{\beta}\right) / T\right]$ in Eq.(2.28) is very small for $g \gg 1$, leading to $\phi_{\alpha} \cong 1$ as in Figs. 1 and 2.

Furthermore, from Eqs. (2.12) and (2.25), the volume fraction $\gamma_{\alpha}$ of phase $\alpha$ is approximated as

$$
\gamma_{\alpha} \cong \bar{n} / n_{\alpha}-e^{-g \Delta \phi} \cong T \bar{n} / G\left(\phi_{\beta}\right)-e^{-g \Delta \phi} .
$$

The above relation is rewritten as

$$
\begin{aligned}
G\left(\phi_{\beta}\right) & \cong T \bar{n} /\left(\gamma_{\alpha}+e^{-g \Delta \phi}\right) \\
& \cong \frac{T \bar{n}\left(1-\phi_{\beta}\right)}{\bar{\phi}-\phi_{\beta}+\left(1-\phi_{\beta}\right) \exp \left[-g\left(1-\phi_{\beta}\right)\right]} .
\end{aligned}
$$

From the first to second line, we have used Eq.(2.11) and replaced $\Delta \phi$ by $1-\phi_{\beta}$. This equation also follows from Eqs.(2.14) and (2.25). It determine $\phi_{\beta}$ and $\gamma_{\alpha} \cong$ $\left(\bar{\phi}-\phi_{\beta}\right) /\left(1-\phi_{\beta}\right)$. We recognize that $G\left(\phi_{\beta}\right)$ increases up to $T \bar{n} e^{g \Delta \phi} \cong T \bar{n} e^{g(1-\bar{\phi})}$ as $\gamma_{\alpha} \rightarrow 0$ or as $\phi_{\beta} \rightarrow \bar{\phi}$. In this limit it follows the marginal relation,

$$
G(\bar{\phi}) \cong T \bar{n} e^{g(1-\bar{\phi})} \quad\left(\gamma_{\alpha} \rightarrow 0\right) .
$$

If $\bar{n}$ is fixed above $n_{\mathrm{p}}$, Eq.(2.32) holds at $\chi=\chi_{\mathrm{p}}$ so that

$$
\chi_{\mathrm{p}} \cong\left[-\ln \bar{\phi}-v_{0} \bar{n} e^{g(1-\bar{\phi})}\right] /(1-\bar{\phi})^{2},
$$

where we use the second line of Eq.(2.26). Notice that the solute density $\bar{n}$ appears in the combination $\bar{n} e^{g(1-\bar{\phi})}$ and its effect of lowering $\chi_{\mathrm{p}}$ is much amplified for $g(1-\phi) \gg 1$ even for very small $\bar{n}$. (For $\bar{n}=0$ the right hand side of Eq.(2.33) is above the coexistence curve.) On the other hand, if $\chi$ is fixed above $\chi_{\mathrm{p}}$, Eq.(2.32) holds at $\bar{n}=n_{\mathrm{p}}$. Thus the minimum solute density $n_{\mathrm{p}}$ is estimated as

$$
n_{\mathrm{p}} \cong e^{-g(1-\bar{\phi})} G(\bar{\phi}) / T,
$$

which is much decreased by the factor $e^{-g(1-\bar{\phi})}$.

In Fig.3, the curves of $\chi_{\mathrm{p}}$ and $n_{\mathrm{p}}$ nearly coincide with the asymptotic formulas (2.33) and (2.34) in the range $\bar{\phi}<0.35$ for $\chi_{\mathrm{p}}$ and in the range $\chi<2$ for $n_{\mathrm{p}}$. They exhibit a minimum at $\bar{\phi} \sim e^{-g} / v_{0} \bar{n} g$ for $\chi_{\mathrm{p}}$ and at $\bar{\phi} \sim$ $g^{-1}$ for $n_{\mathrm{p}}$. For larger $\bar{\phi}>0.35, \chi_{\mathrm{p}}$ nearly coincide with the coexistence curve, indicating disappearance of the precipitation branch. Notice that $n_{\mathrm{p}}$ decreases to zero as $\bar{\phi}$ approaches the coexistence composition $\phi_{\mathrm{cx}}=0.249$ at $\chi=2.2$ (top) and 0.204 at $\chi=2.3$ (bottom), where phase separation occurs without solute.

\section{Theory for small volume fraction of precipitates}

We next construct an analytic theory of precipitation for small volume fraction $\gamma_{\alpha} \ll 1$. We will calculate $F$ in Eq.(2.19) treating $\gamma_{\alpha}$ as an order parameter of the phase transition. Though this theory is applicable only for $\gamma_{\alpha} \ll 1$, it can yield Eq.(2.21) in the asymptotic limit.

In Eq.(2.19) we first expand $\phi_{\beta}$ and $f\left(\phi_{\beta}\right)$ with respect to $\gamma_{\alpha}$ as

$$
\begin{gathered}
\phi_{\beta}=\left(\bar{\phi}-\phi_{\alpha}\right) /\left(1-\gamma_{\alpha}\right) \cong \bar{\phi}+\left(\bar{\phi}-\phi_{\alpha}\right) \gamma_{\alpha} \\
f\left(\phi_{\beta}\right) \cong f(\bar{\phi})+f^{\prime}(\bar{\phi})\left(\bar{\phi}-\phi_{\alpha}\right) \gamma_{\alpha} .
\end{gathered}
$$

Let $F_{0}=V f_{\text {tot }}(\bar{\phi}, \bar{n})$ be the one-phase value of $F$ (at $\left.\gamma_{\alpha}=0\right)$. The increment $\Delta F=F-F_{0}$ is due to precipitate formation and is of the form,

$$
\Delta F / V=\Omega\left(\phi_{\alpha}\right) \gamma_{\alpha}-T \bar{n} \ln \left[1+\Psi\left(\phi_{\alpha}\right) \gamma_{\alpha}\right],
$$

where $\omega_{b}(\phi)$ and $\Psi(\phi)$ are defined by

$$
\begin{aligned}
& \Omega(\phi)=f(\phi)-f(\bar{\phi})-f^{\prime}(\bar{\phi})(\phi-\bar{\phi}), \\
& \Psi(\phi)=\exp [g(\phi-\bar{\phi})]-1-g(\phi-\bar{\phi}),
\end{aligned}
$$




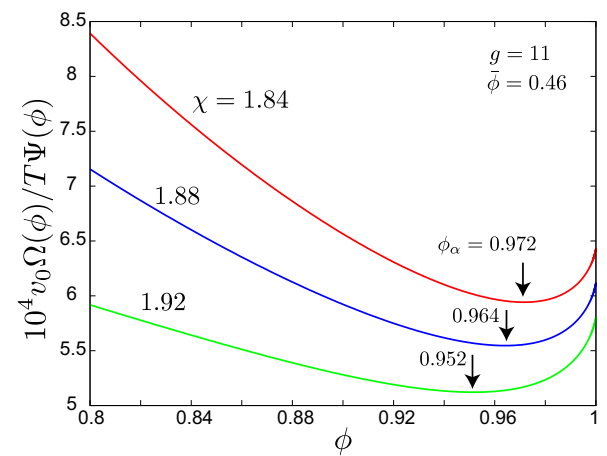

FIG. 5: (Color online) $\Omega(\phi) / \Psi(\phi)$ multiplied by $10^{4} v_{0} / T$ for three values of $\chi$ at $g=11$ and $\bar{\phi}=0.46$. Each curve exhibits a minimum at $\phi=\phi_{\alpha}$, where $\phi_{\alpha}$ is estimated as in Eq.(2.51). Each minimum value is equal to $10^{4} v_{0} n_{\mathrm{p}}$ from Eq.(2.46).
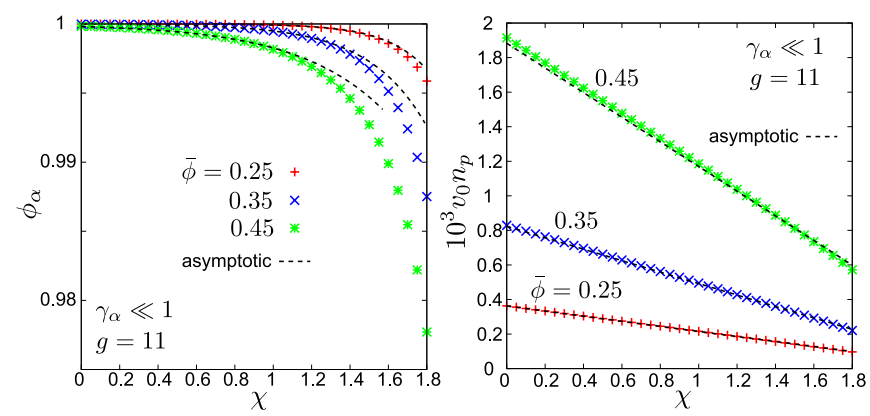

FIG. 6: (Color online) Theoretical $\phi_{\alpha}$ in the range $[0.975,1]$ (left) and $n_{\mathrm{p}}$ multiplied by $10^{3} v_{0}$ (right) for $\bar{\phi}=0.25,0.35$, and 0.45 at $g=11$. These curves are calculated for $\gamma_{\alpha} \ll 1$ from Eqs.(2.45) and (2.46). For these parameters, they only slightly deviate from the asymptotic formulas in Eqs.(2.49) and (2.52). The curves of $n_{\mathrm{p}}$ are nearly linear in $\chi$ from the linear dependence of $G(\bar{\phi})$ on $\chi$.

where $\bar{\phi}$ is treated as a constant and its dependence of $\Omega$ and $\Phi$ are suppressed. In Eq.(2.36) we have not expanded the logarithmic term with respect to $\gamma_{\alpha}$, because the coefficient $\Psi\left(\phi_{\alpha}\right)$ grows strongly as $e^{g\left(\phi_{\alpha}-\bar{\phi}\right)}$ for $g\left(\phi_{\alpha}-\bar{\phi}\right) \gg 1$. For $\chi<2$, the positivity $\Omega\left(\phi_{\alpha}\right)>0$ follows in the region $\bar{\phi}<\phi<\phi_{\alpha}$ since Eq.(2.37) gives

$$
\Omega\left(\phi_{\alpha}\right)=\int_{\bar{\phi}}^{\phi_{\alpha}} d \phi\left(\phi_{\alpha}-\phi\right) f^{\prime \prime}(\phi)
$$

where $f^{\prime \prime}(\phi)=\partial^{2} f / \partial^{2} \phi>0$. Note that if $\phi$ and $\bar{\phi}$ in $\Omega(\phi)$ are replaced by 1 and $\phi_{\beta}$, respectively, $\Omega(\phi)$ becomes identical to $G\left(\phi_{\beta}\right)$ in Eq.(2.26). If use is made of Eq. $(2.4), \Omega(\phi)$ is explicitly written as

$$
\frac{v_{0}}{T} \Omega(\phi)=\phi \ln \left(\frac{\phi}{\bar{\phi}}\right)+(1-\phi) \ln \left(\frac{1-\phi}{1-\bar{\phi}}\right)-\chi(\phi-\bar{\phi})^{2} .
$$

For each given $\bar{\phi}$ and $\bar{n}, \Delta F$ should be minimized with respect to $\gamma_{\alpha}$ and $\phi_{\alpha}$. Here $\phi_{\beta}$ is given by Eq.(2.35).
From Eq.(2.36) its derivative with respect to $\gamma_{\alpha}$ is

$$
\frac{1}{V} \frac{\partial \Delta F}{\partial \gamma_{\alpha}}=\Omega\left(\phi_{\alpha}\right)-\frac{T \bar{n} \Psi\left(\phi_{\alpha}\right)}{1+\Psi\left(\phi_{\alpha}\right) \gamma_{\alpha}} .
$$

The right hand side can vanish only when

$$
Z\left(\phi_{\alpha}\right) \equiv \Omega\left(\phi_{\alpha}\right) / T \bar{n} \Psi\left(\phi_{\alpha}\right)<1 .
$$

If $Z\left(\phi_{\alpha}\right)<1, \gamma_{\alpha}$ is nonvanishing and is written as

$$
\gamma_{\alpha}=T \bar{n} / \Omega\left(\phi_{\alpha}\right)-1 / \Psi\left(\phi_{\alpha}\right) .
$$

For this $\gamma_{\alpha}, \Delta F$ in Eq.(2.36) becomes negative as

$$
\Delta F / V=T \bar{n}\left[1-Z\left(\phi_{\alpha}\right)+\ln Z\left(\phi_{\alpha}\right)\right] .
$$

In the range $0<Z<1$, the function $1-Z+\ln Z$ is negative and decreases with decreasing $Z$. In order to minimize $\Delta F$, we should thus minimize $Z(\phi)$ or the function $\Omega(\phi) / \Psi(\phi)$ at $\phi=\phi_{\alpha}$. The equation to determine $\phi_{\alpha}=\phi_{\alpha}(\phi, \chi)$ is hence given by

$$
\frac{\partial}{\partial \phi_{\alpha}} \frac{\Omega\left(\phi_{\alpha}\right)}{\Psi\left(\phi_{\alpha}\right)}=\frac{\Omega^{\prime}\left(\phi_{\alpha}\right)}{\Psi\left(\phi_{\alpha}\right)}-\frac{\Omega\left(\phi_{\alpha}\right)}{\Psi\left(\phi_{\alpha}\right)^{2}} \Psi^{\prime}\left(\phi_{\alpha}\right)=0,
$$

where $\Omega^{\prime}(\phi)=\partial \Omega(\phi) / \partial \phi$ and $\Psi^{\prime}(\phi)=\partial \Psi(\phi) / \partial \phi$. Note that $\phi_{\alpha}$ is independent of $\bar{n}$ for $\gamma_{\alpha} \ll 1$. In Fig.5, we plot $\Omega(\phi) / \Psi(\phi)$ for three values of $\chi$ at $\bar{\phi}=0.46$ to demonstrate the existence of its minimum at $\phi=\phi_{\alpha}$. In the left panel of Fig.6, we show $\phi_{\alpha}$ calculated from the above equation.

In terms of the above $\phi_{\alpha}$ (dependent on $\bar{\phi}$ and $\chi$ ), the minimum solute density $n_{\mathrm{p}}$ is obtained from the condition $Z\left(\phi_{\alpha}\right)=1$ as

$$
n_{\mathrm{p}}=\Omega\left(\phi_{\alpha}\right) / T \Psi\left(\phi_{\alpha}\right),
$$

which is a function of $\bar{\phi}$ and $\chi$. Then $Z\left(\phi_{\alpha}\right)=n_{\mathrm{p}} / \bar{n}$. The condition $Z\left(\phi_{\alpha}\right)<1$ yields $\bar{n}>n_{\mathrm{p}}$, as ought to be the case, and $\gamma_{\alpha}$ is also expressed as

$$
\gamma_{\alpha}=\left(\bar{n} / n_{\mathrm{p}}-1\right) / \Psi\left(\phi_{\alpha}\right) .
$$

In the right panel of Fig.6, we show $n_{\mathrm{p}}$ calculated from Eq.(2.46) using the data of $\phi_{\alpha}$ in the left panel.

After $\phi_{\alpha}$ and $n_{\mathrm{p}}$ have been determined, Eq.(2.47) indicates that an arbitrary value can be assigned to $\bar{n}$ in the range $0<\bar{n} / n_{\mathrm{p}}-1 \ll \Psi\left(\phi_{\alpha}\right)$. Furthermore, by decreasing $\chi$ at fixed $\bar{n}$ and $\bar{\phi}$, we can make $Z\left(\phi_{\alpha}\right)$ approach unity, where $\chi \rightarrow \chi_{\mathrm{p}}=\chi_{\mathrm{p}}(\bar{\phi}, \bar{n})$ in Eq.(2.21). Using Eq.(2.40) we may express $\chi_{\mathrm{p}}$ as

$$
\begin{aligned}
\chi_{\mathrm{p}}= & {\left[\phi_{\alpha} \ln \frac{\phi_{\alpha}}{\bar{\phi}}+\left(1-\phi_{\alpha}\right) \ln \frac{1-\phi_{\alpha}}{1-\bar{\phi}}\right] /\left(\phi_{\alpha}-\bar{\phi}\right)^{2} } \\
& -v_{0} \bar{n} \Psi\left(\phi_{\alpha}\right) /\left(\phi_{\alpha}-\bar{\phi}\right)^{2} .
\end{aligned}
$$

The relations (2.46) and (2.48) for the lower bounds are exact in our model and are consistent with the asymptotic ones (2.33) and (2.34) in the limit of $\phi_{\alpha} \cong 1$ and $\Psi\left(\phi_{\alpha}\right) \cong e^{g(1-\phi)} \gg 1$. 
We now give analysis of the function $\Omega(\phi) / \Psi(\phi)$ in the range $\phi<\bar{\phi}$. If $\phi$ is not very close to unity, $\Omega(\phi) / \Psi(\phi)$ decreases rapidly with increasing $\phi$, because $1 / \Psi(\phi) \propto e^{-g \phi}$ decreases rapidly. However, as $\phi \rightarrow 1$, the logarithmic term in $f(\phi)$ eventually comes into play. To show this, we approximate $\Omega(\phi)$ for $\phi \cong 1$ as

$$
\Omega(\phi) \cong G(\bar{\phi})+\frac{T}{v_{0}}(1-\phi)\left[\ln \frac{1-\phi}{A_{0}}-1\right],
$$

where $G(\phi)$ is defined in Eq.(2.26) and $\ln \left(A_{0}\right)=-\chi-$ $v_{0} f^{\prime}(\bar{\phi}) / T_{-}$. The coefficient $A_{\beta}$ in Eq. $(2.29)$ tends to $A_{0}$ as $\phi_{\beta} \rightarrow \bar{\phi}$. The derivative of $\Omega(\phi) / \Psi(\phi)$ with respect to $\phi$ is thus estimated as

$$
\frac{d}{d \phi} \frac{\Omega(\phi)}{\Psi(\phi)} \cong\left[-g G(\bar{\phi})-\frac{T}{v_{0}} \ln \frac{1-\phi}{A_{0}}\right] e^{-g(\phi-\bar{\phi})} .
$$

In this derivative, the logarithmic term grows weakly but can balance the solvation term $(\propto g)$ as $\phi \rightarrow 1$, so that

$$
1-\phi_{\alpha} \cong A_{0} \exp \left(-g v_{0} G(\bar{\phi}) / T\right)
$$

in accord with Eq.(2.28). The asymptotic form of $n_{\mathrm{p}}$ is written as in Eq.(2.34) if we set $\Omega\left(\phi_{\alpha}\right) \cong G(\bar{\phi})$ and $\Psi(\phi) \cong e^{g \Delta \phi}$ in Eq.(2.46).

\section{INHOMOGENEOUS COMPOSITION PROFILES}

Here, still neglecting the charge effect, we examine the surface tension, the stability of a spherical droplet, and the surface adsorption near a boundary wall. These problems stem from the solvation-induced phase separation.

\section{A. Gradient free energy and surface tension}

Including the gradient free energy we assume the following simple form for the total free energy,

$$
F=\int d \boldsymbol{r}\left[f_{\mathrm{tot}}(\phi, n)+\frac{C}{2}|\nabla \phi|^{2}\right],
$$

where $f_{\text {tot }}$ is given by Eq.(2.3) and $C$ is assumed to be a positive constant. We suppose a planar interface at $z=0$ varying along the $z$ axis and separating two phases $\alpha$ and $\beta$. Then $\phi=\phi(z)$ and $n=n(z)$ are functions of $z$ with $\phi(-\infty)=\phi_{\alpha}$ and $\phi(\infty)=\phi_{\beta}$. In equilibrium, the functional derivatives of $F$ with respect to $\phi$ and $n$ are constant in space. Therefore,

$$
h=\delta F / \delta \phi=f^{\prime}(\phi)-T g n-C \phi^{\prime \prime}=\text { constant },
$$

where $\phi^{\prime \prime}=d^{2} \phi / d z^{2}$. Since $\mu=\delta F / \delta n=\partial f_{\text {tota }} / \partial n$ is given by Eq.(2.7), the solute density depends on $\phi$ as

$$
n(z)=A_{0} \exp [g \phi(z)]
$$

where the coefficient $A_{0}$ is determined by Eq.(2.9) at fixed $\bar{n}=\langle n\rangle$.

We multiply Eq.(3.2) by $\phi^{\prime}=d \phi / d z$ and integrate it over $z$ to obtain

$$
\Delta \omega(\phi)=\omega(\phi)-\omega\left(\phi_{\beta}\right)=\frac{1}{2} C\left(\phi^{\prime}\right)^{2},
$$

where $\omega(\phi)=f-T n-h \phi$ is the grand potential density in Eq.(2.16) satisfying $\omega\left(\phi_{\alpha}\right)=\omega\left(\phi_{\beta}\right)$ as in Eq.(2.17). From Eq.(2.15) and the second line of Eq.(2.16), the difference $\Delta \omega(\phi)$ is written as

$$
\begin{gathered}
\Delta \omega(\phi)=f(\phi)-f\left(\phi_{\beta}\right)-f^{\prime}\left(\phi_{\beta}\right)\left(\phi-\phi_{\beta}\right) \\
-T n_{\beta}\left[e^{g\left(\phi-\phi_{\beta}\right)}-g\left(\phi-\phi_{\beta}\right)-1\right] .
\end{gathered}
$$

It is worth noting that the limit $\gamma_{\alpha} \rightarrow 0$ can be attained at $\bar{n}=n_{\mathrm{p}}$. From Eq.(3.5) we find

$$
\lim _{\gamma_{\alpha} \rightarrow 0} \Delta \omega(\phi)=\Omega(\phi)-\left[\Omega\left(\phi_{\alpha}\right) / \Psi\left(\phi_{\alpha}\right)\right] \Psi(\phi),
$$

where $\Omega(\phi)$ and $\Psi(\phi)$ are defined in Eqs. (2.37) and (2.38). From Eq.(2.45) the derivative of the left hand side of Eq.(3.6) with respect to $\phi$ vanishes at $\phi=\phi_{\alpha}$. There is no singular behavior in the interface profile even in the limit $\gamma_{\alpha} \rightarrow 0$.

The relation $\Delta \omega(\phi) \cong \omega^{\prime \prime}\left(\phi_{\beta}\right)\left(\phi-\phi_{\beta}\right)^{2} / 2$ for $\phi \cong \phi_{\beta}$ follows directly from Eq.(3.5). The $\Delta \omega(\phi)$ also behaves as $\omega^{\prime \prime}\left(\phi_{\alpha}\right)\left(\phi-\phi_{\alpha}\right)^{2} / 2$ for $\phi \cong \phi_{\alpha}$ from Eqs.(2.15) and (2.17). Here $\omega^{\prime \prime}(\phi)=f^{\prime \prime}(\phi)-T g^{2} n(\phi)$ is the second derivative of $\Delta \omega(\phi)$ with respect to $\phi$ and the correlation lengths $\xi_{\alpha}$ and $\xi_{\beta}$ in the bulk two phases are given by

$$
\begin{aligned}
& C \xi_{\alpha}^{-2}=f^{\prime \prime}\left(\phi_{\alpha}\right)-T g^{2} n_{\alpha}, \\
& C \xi_{\beta}^{-2}=f^{\prime \prime}\left(\phi_{\beta}\right)-T g^{2} n_{\beta},
\end{aligned}
$$

in terms of which $\phi(z)-\phi_{\alpha} \propto e^{-|z| / \xi_{\alpha}}$ for $z<-\xi_{\alpha}$ and $\phi(z)-\phi_{\beta} \propto e^{-z / \xi_{\beta}}$ for $z>\xi_{\beta}$. The right hand sides of Eq.(3.7) should be positive for the stability of the bulk two phases. However, since $f^{\prime \prime}\left(\phi_{\alpha}\right) \sim T / v_{0}\left(1-\phi_{\alpha}\right), \xi_{\alpha}$ becomes even shorter than $a=v_{0}^{1 / 3}$ for $\phi_{\alpha} \cong 1$ and $C \sim T / a$. Thus the composition varies too steeply in phase $\alpha$ in the present gradient theory. However, we shall see (around Eq.(3.11) below) that the surface tension is mainly determined by the composition variation in the phase $\beta$ side of an interface.

In $\Delta \omega(\phi)$ in Eq.(3.5), the last term in the second line grows as $\exp \left[g\left(\phi-\phi_{\beta}\right)\right]$ for $\phi-\phi_{\beta} \gg 1 / g$ and can balance the other terms with increasing $\phi$ even for very small $n_{\beta}$. In Fig.7, we illustrates this behavior by plotting the sum of the first three terms and the minus of the last term in the right hand side of Eq.(3.5) separately. That is, we write $\Delta \omega(\phi)=\Delta \omega_{0}(\phi)-\omega_{\text {sol }}(\phi)$ with

$$
\begin{aligned}
& \Delta \omega_{0}(\phi)=f(\phi)-f\left(\phi_{\beta}\right)-f^{\prime}\left(\phi_{\beta}\right)\left(\phi-\phi_{\beta}\right), \\
& \omega_{\text {sol }}(\phi)=T n_{\beta}\left[e^{g\left(\phi-\phi_{\beta}\right)}-g\left(\phi-\phi_{\beta}\right)-1\right] .
\end{aligned}
$$

The $\Delta \omega_{0}(\phi)$ is the grand potential difference without solute. As a function of $\phi$, it increases monotonically for 

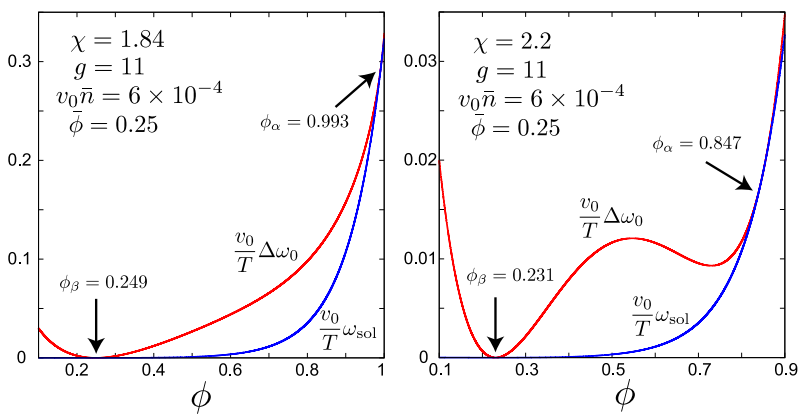

FIG. 7: $\Delta \omega_{0}(\phi)$ and $\omega_{\text {sol }}(\phi)$ in eq. (2.59) in units of $T / v_{0}$ for $\chi=1.84$ (left) and 2.2 (right), where $g=11, \bar{\phi}=0.25$, and $v_{0} \bar{n}=6 \times 10^{-4}$. The solute part $\omega_{\text {sol }}(\phi)$ is negligibly small for $\phi \lesssim 0.6$ here, but it grows abruptly for larger $\phi$ and becomes equal to $\Delta \omega_{0}(\phi)$ at $\phi=\phi_{\alpha}$. This ensures the existence of an interface connecting phases $\alpha$ and $\beta$.
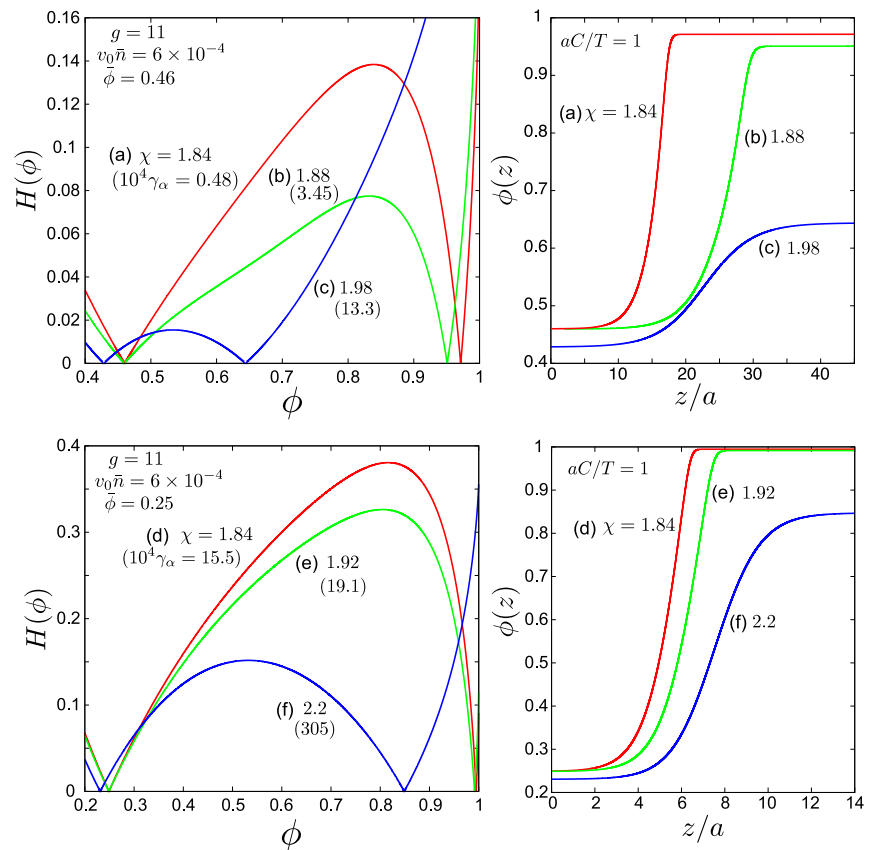

FIG. 8: Left: $H(\phi)$ in Eq.(2.56) vs $\phi$, which vanishes at $\phi=\phi_{\alpha}$ and $\phi_{\beta}$. Right: $\phi(z)$ vs $z / a$, where $a=v_{0}^{1 / 3}$ and $C=T / a$. Here $g=11, \bar{\phi}=0.25$, and $v_{0} \bar{n}=6 \times 10^{-4}$. In the upper plates three curves correspond to (a) $\chi=1.98$, (b) 1.88 , and (c) 1.84 , while in the lower plates we set (d) $\chi=2.2$, (e) 1.92, and (f) 1.84. In the parentheses the volume fraction $\gamma_{\alpha}$ multiplied by $10^{4}$ is written for each curve (left).

$\chi<2$ and exhibits positive extrema for $\chi>2$ (with $\phi_{\beta}$ being outside the coexistence curve).

In the Ginzburg-Landau scheme, the surface tension $\sigma$ is given by the $z$ integration of the (generalized) grand potential density $\Delta \omega(\phi)+C\left(\phi^{\prime}\right)^{2} / 2$ including the gradi- ent contribution. Use of Eq.(3.4) yields

$$
\sigma=\int d z C\left(\phi^{\prime}\right)^{2}=\sqrt{C T / v_{0}} \int_{\phi_{\beta}}^{\phi_{\alpha}} d \phi H(\phi),
$$

where we define the dimensionless function,

$$
H(\phi)=\left(2 v_{0} / T\right)^{1 / 2}\left[\omega(\phi)-\omega\left(\phi_{\beta}\right)\right]^{1 / 2} .
$$

In our theory, $C$ is an arbitrary constant and $\sigma \propto C^{1 / 2}$. In Fig.8, we plot $H(\phi)$ and $\phi(z)$ for (a) $\chi=1.84$, (b) 1.88 , and (c) 1.98 with $\bar{\phi}=0.46$ in the upper plates and for (d) $\chi=1.84$, (e) 1.92 , and (f) 2.2 with $\bar{\phi}=0.25$ in the lower plates. Here $g=11, v_{0} \bar{n}=6 \times 10^{-4}$, and $C=T / a$. The values $\left(a^{2} \sigma / T, \bar{n} / n_{\mathrm{p}}\right)$ in these cases are (a) $(0.044,1.01),(\mathrm{b})(0.024,1.17),(\mathrm{c})(0.0021,2.25),(\mathrm{d})$ $(0.19,6.6),(\mathrm{e})(0.16,7.7)$, and (f) $(0.064, \infty)$. In the case (c), the system is close to the solvent criticality and $\xi_{\beta}$ is relatively long. In the case (f), $\bar{\phi}$ is slightly larger than the composition 0.249 on the oil-rich branch of the coexistence curve without solute, so phase separation occurs even without solute or $n_{\mathrm{p}}=0$. The shape of $H(\phi)$ in Fig.8 is roughly triangular except (f), so we obtain a simple estimate,

$$
\sigma \sim C(\Delta \phi)^{2} / \xi_{\beta} .
$$

Since $\xi_{\alpha}$ is considerably shorter than $\xi_{\beta}$, we may approximate the interface profile as

$$
\phi(z)-\phi_{\beta} \sim \Delta \phi \exp \left(-z / \xi_{\beta}\right),
$$

in the region $0<z \lesssim \xi_{\beta}$, neglecting the variation in the region $-\xi_{\alpha} \lesssim z<0$. Substitution of Eq.(3.12) into Eq.(3.9) yields Eq,(3.11). The behaviors (3.11) and (3.12) hold even in the limit $\gamma_{\alpha} \rightarrow 0$.

\section{B. Discontinuous appearance of a droplet due to surface tension: minimum droplet radius $R_{\mathrm{m}}$}

As an example, we suppose a single spherical droplet of phase $\alpha$ suspended in phase $\beta$ in equilibrium, accounting for the effect of the surface tension $\sigma$. The droplet radius $R$ is so small that the following condition holds:

$$
u \equiv \Psi\left(\phi_{\alpha}\right) \gamma_{\alpha} \ll 1,
$$

where $\gamma_{\alpha}=4 \pi R^{3} / 3 V$. and $\Psi\left(\phi_{\alpha}\right) \sim e^{g(1-\bar{\phi})}$. We add the surface free energy $4 \pi \sigma R^{2}$ to $\Delta F$ in Eq.(2.36) and expand the logarithmic factor there as $\ln (1+u) \cong u-u^{2} / 2$. The resultant total free energy reads

$$
\Delta F_{\text {tot }}=V\left(-w \gamma_{\alpha}+\frac{1}{2} T \bar{n} u^{2}\right)+4 \pi \sigma R^{2},
$$

up to second order in $\gamma_{\alpha}$. The coefficient $w$ is defined by

$$
\begin{aligned}
w & =-\Omega\left(\phi_{\alpha}\right)+T \bar{n} \Psi\left(\phi_{\alpha}\right) \\
& =\Omega\left(\phi_{\alpha}\right)\left(\bar{n} / n_{\mathrm{p}}-1\right) \\
& =T v_{0}^{-1}\left(\phi_{\alpha}-\bar{\phi}\right)^{2}\left(\chi-\chi_{\mathrm{p}}\right),
\end{aligned}
$$


where $n_{\mathrm{p}}$ in the second line is the minimum solute density in Eq. (2.46) and $\chi_{\mathrm{p}}$ in the third line is the minimum interaction parameter in Eq.(2.48). A droplet can exit only for $w>0$, which means $\bar{n}>n_{\mathrm{p}}$ or $\chi>\chi_{\mathrm{p}}$. It is convenient to introduce two characteristic lengths by

$$
\begin{aligned}
R_{c} & =2 \sigma / w, \\
R_{\mathrm{m}} & =(3 \sigma V / 2 \pi T \bar{n})^{1 / 4} \Psi\left(\phi_{\alpha}\right)^{-1 / 2},
\end{aligned}
$$

in terms of which $\Delta F_{\text {tot }}$ in Eq.(3.14) is rewritten as

$$
\Delta F_{\text {tot }} / 4 \pi \sigma=R^{2}-2 R^{3} / 3 R_{c}+R^{6} / 3 R_{\mathrm{m}}^{4},
$$

For $\sigma \sim T / a^{2}$ we roughly estimate

$$
\begin{aligned}
R_{c} & \sim a /\left(\bar{n} / n_{\mathrm{p}}-1\right) \sim a /\left(\chi-\chi_{\mathrm{p}}\right) \\
R_{\mathrm{m}} & \sim\left(n_{\mathrm{p}} / \bar{n}\right)^{1 / 4}(V a)^{1 / 4} e^{-g(1-\bar{\phi}) / 4} .
\end{aligned}
$$

If $\bar{n}$ (or $\chi$ ) is increased above $n_{\mathrm{p}}$ (or $\chi_{\mathrm{p}}$ ), $R_{\mathrm{m}}$ soon exceeds $R_{c}$ for large $V$, despite the reducing factor $e^{-g(1-\bar{\phi}) / 4}$.

We should minimize $\Delta F_{\text {tot }}$ in Eq.(3.20) as a function of $R$. For $R>0$ we require $\Delta F_{\text {tot }}<0$ and $\partial \Delta F_{\text {tot }} / \partial R=0$. The latter condition is written as

$$
1-R / R_{c}+R^{4} / R_{\mathrm{m}}^{4}=0,
$$

under which $\Delta F_{\text {tot }}=4 \pi R^{2}\left(2-R / R_{c}\right) / 3<0$, so $R>2 R_{c}$ is needed. This inequality can be satisfied only for

$$
R_{c}<R_{\mathrm{m}} / 2 \quad \text { or } \quad w>4 \sigma / R_{\mathrm{m}}, .
$$

which will be evident in Fig.18 below. If the above condition is not satisfied, there is no equilibrium droplet. As $R_{\mathrm{m}} / R_{c} \rightarrow 2$, we have $R \rightarrow R_{\mathrm{m}}$. Here $u$ in Eq.(3.13) is of order $\left(n_{\mathrm{p}} / \bar{n}\right)^{3 / 4}\left[a^{3} e^{g(1-\bar{\phi})} / V\right]^{1 / 4}$ and can be much smaller than unity for large $V$. Thus $R_{\mathrm{m}}$ is the minimum equilibrium droplet radius in the presence of the surface tension. For $R_{\mathrm{m}} / R_{c} \gg 1$, the surface free energy becomes negligible and

$$
R \cong\left(R_{\mathrm{m}}^{4} / R_{c}\right)^{1 / 3}=\left[3 V w / 4 \pi \bar{n} \Psi\left(\phi_{\alpha}\right)^{2}\right]^{1 / 3} .
$$

We also note that the transition occurs for $\bar{n} / n_{\mathrm{p}}-1 \gtrsim$ $a / R_{\mathrm{m}}$ from Eq.(3.16) or for $(1-\bar{\phi})^{2}\left(\chi-\chi_{\mathrm{p}}\right) \gtrsim a / R_{\mathrm{m}}$ from Eq.(3.17).

In Fig.9, we plot $\Delta F_{\text {tot }}=\Delta F+4 \pi \sigma R^{2}$ as a function of $R$ in the spherically symmetric geometry, where $\bar{\phi}=$ $0.35, v_{0} \bar{n}=6 \times 10^{-6}$, and $g=11$. The cell volume is $V=4 \pi L^{3} / 3 \cong 0.9 \times 10^{9} v_{0}$ with $L=600 a$. Here we use Eq.(2.36) for $\Delta F$ and not the expansion form of $\Delta F$ in Eq.(3.14). For the three curves in Fig.9, the parameter $u$ in Eq.(3.13) is surely smaller than unity and Eq.(3.14) or Eq.(3.20) is a good approximation. In fact, at the minimum position of the curve (b) in Fig.9, we have $R=18.23 a, \sigma=0.231 T / a^{2}$, and $\Omega=0.706 T / v_{0}$, while $R_{\mathrm{m}}=17.89 a$ from Eq.(2.70) in this case. In the next section, we will present simulation results in the same situation for hydrophilic ions.

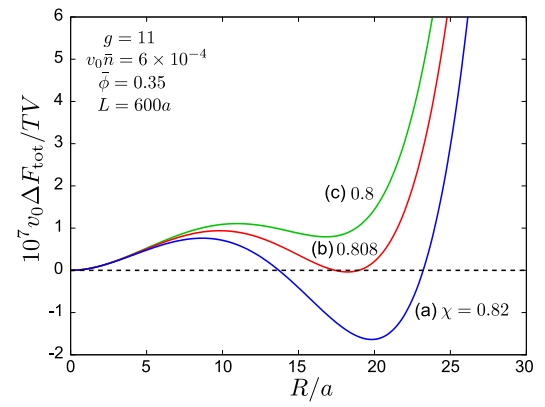

FIG. 9: (Color online) $\Delta F_{\text {tot }}$ including the surface free energy vs $R / a$ for (a) $\chi=0.82$, (b) 0.808 , and (c) 0.8 from below. At the minimum with positive $R$, the droplet is stable for (a), marginal for (b), and metastable for (c), while $\bar{n} / n_{\mathrm{p}}=1.080$ for (a), 1.073 for (b), and 1.067 for (c). Here $R=18.23 a$ at the minimum for $(b)$.

\section{Solvation-induced prewetting transition}

We note that Eq.(3.14) can also be used to examine the composition profile $\phi(z)$ near a boundary wall at $z=0$. Here, slightly below the precipitation curve $\chi=\chi_{\mathrm{p}}$ (or $\bar{n}=n_{\mathrm{D}}$ ), we may predict a first-order prewetting transition [36, 37] at $\chi=\chi_{\operatorname{tr}}\left(<\chi_{p}\right)$ with increasing $\chi$, where the surface adsorption changes discontinuously. This result is related to the experiment by Beaglehole [21].

Far from the wall, we assume that $\phi(z)$ and $n(z)$ tend to $\bar{\phi}$ and $\bar{n}$, respectively. Then Eq.(3.4) holds with

$$
\Delta \omega(\phi)=\Omega(\phi)-T \bar{n} \Phi(\phi),
$$

where $\Omega(\phi)$ and $\Phi(\phi)$ are defined by Eqs. (2.37) and (2.38). The previous $\Delta \Omega(\phi)$ in Eqs.(3.4) and (3.5) becomes the above $\Delta \Omega(\phi)$ if $\phi_{\beta}$ and $n_{\beta}$ there are replaced by $\bar{\phi}$ and $\bar{n}$, respectively. Below the precipitation curve $\chi=\chi_{\mathrm{p}}, \Delta \omega(\phi)$ is positive for $\phi>\bar{\phi}$ and again approaches zero at $\phi \cong \phi_{\alpha}$ as $\chi \rightarrow \chi_{\mathrm{p}}$. See Fig.7 for its behavior. Assuming $\phi^{\prime}(z)=d \phi(z) / d z<0$, we rewrite Eq.(3.4) as

$$
\frac{d \phi}{d z}=-\sqrt{2 \Delta \omega(\phi) / C} .
$$

In addition to the bulk free energy $F$ in Eq.(3.1), we assume the surface free energy,

$$
F_{s}=-\int d S \alpha_{\mathrm{w}} \phi=-S \alpha_{\mathrm{w}} \phi_{s}
$$

where $\int d S$ is the surface integral at $z=0, S$ is the surface area, $\phi_{s}=\phi(0)$, and $\alpha_{w}$ is a parameter arising from the short-range interaction between the solvent and the surface. Here $\alpha_{w}>0$ if the boundary wall is hydrophilic and the solute is hydrophilic. The boundary condition of $\phi(z)$ at $z=$ is given by

$$
C \phi^{\prime}(0)=-\alpha_{\mathrm{w}} .
$$



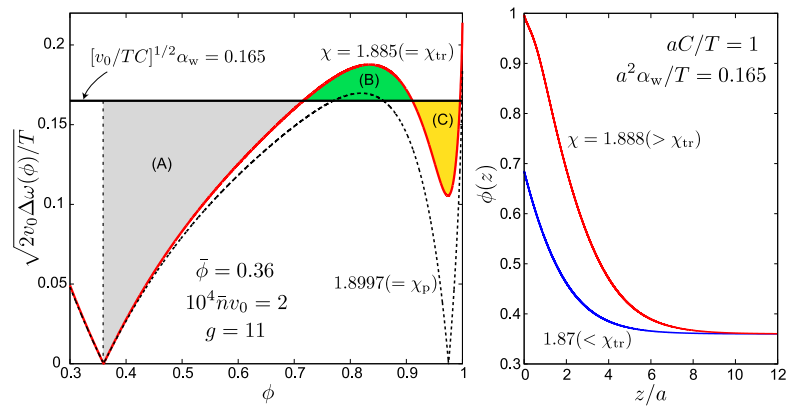

FIG. 10: (Color online) Left: $\sqrt{2 v_{0} \Delta \omega(\phi) / T}$ vs $\phi$ with $\bar{\phi}=$ 0.36 and $v_{0} \bar{n}=2 \times 10^{-4}$, where $\Delta \omega(\phi)$ is defined in Eq.(3.25). For $\alpha_{\mathrm{w}}=0.165\left(C T / v_{0}\right)^{1 / 2}$, a first-order prewetting transition at a wall occurs at $\chi=\chi_{\mathrm{tr}}=1.885$, where the areas $(\mathrm{B})$ and $(\mathrm{C})$ coincide. Bulk two-phase coexistence is realized for $\chi>\chi_{\mathrm{p}}=1.8997$. Right: $\phi(z)$ for $C=T / a$ near the wall before and after the prewetting transition.

Then $\sqrt{2 C \Delta \omega(\phi)}=\alpha_{\mathrm{w}}$ at $\phi=\phi_{s}$. The surface free energy density per unit area is then written as [36, 37]

$$
\begin{aligned}
\mathcal{F}_{\mathrm{ad}} & =\int_{0}^{\infty} d z[\Omega(\phi)-T \bar{n} \Psi(\phi)]-\alpha_{\mathrm{w}} \phi_{s} \\
& =\int_{\bar{\phi}}^{\phi_{s}} d \phi\left[\sqrt{2 C \Delta \omega(\phi)}-\alpha_{\mathrm{w}}\right]-\alpha_{\mathrm{w}} \bar{\phi} .
\end{aligned}
$$

Without solute, the composition increases by $\alpha_{w} \xi / C$ on the surface for $\left|\alpha_{w}\right| \ll T / a^{2}$, where $\xi$ is the correlation length. However, in the presence of solute with $g \gg 1$, the adsorption on the surface can be strong with $\phi_{s}$ close to unity for $g \alpha_{w} \xi / C \gtrsim 1$ even when $\bar{n}$ is very small. In such cases, $w_{\text {sol }}$ and $\Delta w_{0}$ in Eq.(2.59) are of the same order on the surface (see Fig.7). In the left panel of Fig.10, $\sqrt{2 v_{0} \Delta \omega(\phi) / T}$ vs $\phi$ is displayed for $\chi=\chi_{\mathrm{tr}}=$ 1.885 and $\chi_{\mathrm{p}}=1.8997$, where $\bar{\phi}=0.36, \bar{n}=2 \times 10^{-4}$, and $\alpha_{\mathrm{w}}=0.165\left(C T / v_{0}\right)^{1 / 2}$. It demonstrates the presence of a first-order prewetting transition. In the right panel of Fig.10, the composition profile $\phi(z)$ is shown slightly before and after the transition.

\section{HYDROPHILIC SALT}

We now treat aqueous mixtures containing a small amount of hydrophilic monovalent salt. We shall see that the charge imbalance appears only near the interface as an electric double layer. In the statics of the present problem, the role of the electrostatic interaction is thus to shift the surface tension slightly (see the last paragraph of Sec.IVA) 23]. In the dynamics, on the other hand, electric double layers around the droplet surfaces should suppress fusion of approaching droplets. Such dynamical aspects are beyond the scope of this paper and should be studied in future.

\section{A. Preferential ion solvation}

We write the cation density as $n_{1}$ and the anion density as $n_{2}$. Their total amounts are fixed as

$$
\int d \boldsymbol{r} n_{1}=\int d \boldsymbol{r} n_{2}=V \bar{n} / 2 .
$$

These densities both tend to $n_{\alpha} / 2$ or $n_{\beta} / 2$ in the bulk regions of phase $\alpha$ or $\beta$ due to the charge balance. The electric charge density $e\left(n_{1}-n_{2}\right)$ gives rise to the electric potential $\Phi$ satisfying the Poisson equation,

$$
\nabla \cdot \varepsilon \nabla \Phi=-4 \pi e\left(n_{1}-n_{2}\right) .
$$

The dielectric constant $\varepsilon(\phi)$ can depend on $\phi$ and has been assumed to be of the form,

$$
\varepsilon(\phi)=\varepsilon_{0}+\varepsilon_{1} \phi .
$$

Neglecting the image interaction [23, 34], we assume the total free energy $F$ in the following form, 23]

$$
\begin{aligned}
F & =\int d \boldsymbol{r}\left[f(\phi)+\frac{1}{2} C|\nabla \phi|^{2}+\frac{\varepsilon}{8 \pi}|\nabla \Phi|^{2}\right. \\
& \left.+T \sum_{i=1,2}\left[n_{i} \ln \left(n_{i} \lambda_{i}^{3}\right)-g_{i} \phi n_{i}\right]\right]
\end{aligned}
$$

where the third term is the electrostatic contribution and the terms proportional to $g_{i}$ represent the solvation interaction among the ions and the solvent composition. The $\lambda_{1}$ and $\lambda_{2}$ are the thermal de Broglie lengths of the two ion species.

Let us explain the solvation terms in more detail. The ion chemical potentials due to solvation in aqueous mixtures strongly depend on the ambient solvent composition $\phi[23,33]$. We write them as $\mu_{\text {sol }}^{i}(\phi)$, where $i$ represents the ion species. We assume the linear form,

$$
\mu_{\mathrm{sol}}^{i}(\phi)=\mu_{0}^{i}-T g_{i} \phi,
$$

where the first terms are irrelevant constants. This linear dependence is adopted to gain the physical consequences in the simplest manner and should not be taken too seriously. In aqueous solutions, $g_{i}>0$ for hydrophilic ions and $g_{i}<0$ for hydrophobic ions. When two phases $\alpha$ and $\beta$ coexist, the difference

$$
\Delta \mu_{\mathrm{sol}}^{i}=\mu_{\mathrm{sol}}^{i}\left(\phi_{\beta}\right)-\mu_{\mathrm{sol}}^{i}\left(\phi_{\alpha}\right)
$$

is called the Gibbs transfer free energy in electrochemistry (usually measured in units of $\mathrm{kJ} /$ per mole), leading to an electric potential difference across an interface, called the Galvani potential difference. If we assume the linear form (3.5), it is expressed as

$$
\Delta \mu_{\mathrm{sol}}^{i}=T g_{i} \Delta \phi .
$$

For example, in water-nitrobenzene(NB) at $300 \mathrm{~K}$, it can be estimated as $17 T$ for $\mathrm{Na}^{+}$and as $19 T$ for $\mathrm{Cl}^{-}$per 
ion. In this mixture, the two phases are strongly segregated $(\Delta \phi \cong 1)$ and we estimate $g_{i}=15-20$ for small hydrophilic monovalent ions. In heavy water-trimethylpyridine(3MP), the selective solvation has been found to induce mesoscopic phases with periodic structures for antagonistic salt. The Gibbs transfer free energies of hydrophilic ions for water-3MP should not be larger than or at least of the same order as those for water- $\mathrm{NB}$, in view of the fact that the dielectric constants of $3 \mathrm{MP}$ and NB are 9 and 35, respectively.

We may calculate the composition and ion profiles from the homogeneity of the chemical potentials $h=\delta F / \delta \phi$ and $\mu_{i}=\delta F / \delta n_{i}$, where

$$
h=f^{\prime}-C \nabla^{2} \phi-\frac{\varepsilon_{1}}{8 \pi}|\nabla \Phi|^{2}-\sum_{i} g_{i} n_{i} .
$$

If $\mu_{i}$ are homogeneous, we obtain the modified PoissonBoltzmann relations,

$$
n_{i}=\frac{1}{2} n_{\beta} \exp \left[g_{i}\left(\phi-\phi_{\beta}\right) \mp e\left(\Phi-\Phi_{\beta}\right) / T\right],
$$

where - is for $i=1$ and + is for $i=2$. The potential $\Phi$ tends to constants $\Phi_{\alpha}$ and $\Phi_{\beta}$ in the bulk regions. From the charge neutrality in the bulk regions, the potential difference between the two phases is given by

$$
\Delta \Phi=\Phi_{\alpha}-\Phi_{\beta}=T\left(g_{1}-g_{2}\right) \Delta \phi / 2 e .
$$

Then the ion densities in the two phases satisfy

$$
n_{\alpha} / n_{\beta}=\exp \left[\left(g_{1}+g_{2}\right) \Delta \phi / 2\right] .
$$

The charge density appears only near the interface for $g_{1} \neq g_{2}$ forming an electric double layer (see Fig.11). As a result, the bulk phase relations in Eqs.(2.8)-(2.18) for neutral particles still hold for ions with the correspondence relations,

$$
n=n_{1}+n_{2}, \quad g=\left(g_{1}+g_{2}\right) / 2 .
$$

For $g_{1}=g_{2}$ we have $n_{1}=n_{2}=n / 2$.

The thickness of the electric double layer is of the order of the Debye screening length, $\lambda_{\alpha}$ in the phase $\alpha$ side and $\lambda_{\beta}$ in the phase $\beta$ side, where

$$
\lambda_{K}=\left(\varepsilon_{K} T / 4 \pi n_{K} e^{2}\right)^{1 / 2},
$$

where $K=\alpha$ or $\beta$. For hydrophilic ions, $\lambda_{\alpha} \ll \lambda_{\beta}$ holds from $n_{\alpha} \gg n_{\beta}$ and $\lambda_{\alpha}$ is longer than the interface thickness except close to the solvent criticality. We assume that the domain size is much longer than $\lambda_{\beta}$.

We found that the surface tension of an interface of a precipitated domain is slightly decreased by the electric double layer appearing for $g_{1} \neq g_{2}$. In fact, for $v_{0} \bar{n} \sim$ $10^{-3}$, it is larger by a few $\%$ for $g_{1}=15$ and $g_{2}=7$ than for $g_{1}=g_{2}=11$, where the other parameters are common.

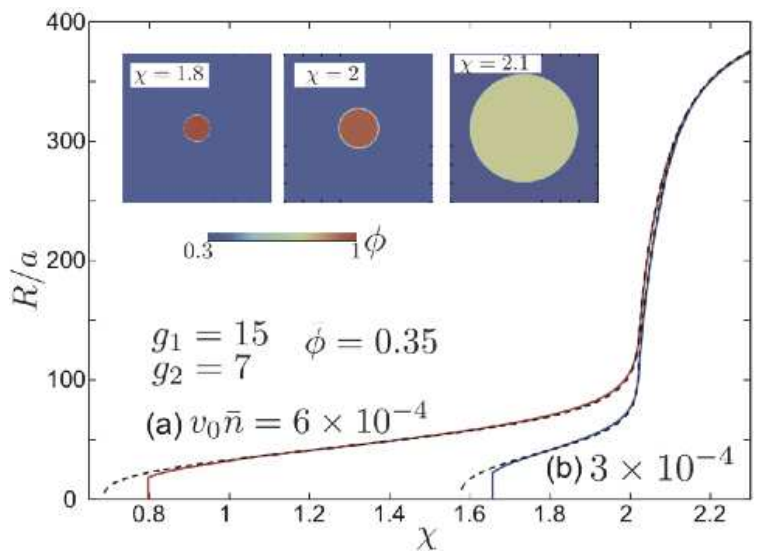

FIG. 11: (Color online) $R / a$ vs $\chi$ (bold line) with hydrophilic ions calculated from the free energy (4.4) for (a) $v_{0} \bar{n}=3 \times$ $10^{-4}$ (blue line) and (b) $6 \times 10^{-4}$ (red line). Plotted also are theoretical curves from the approximate free energy (2.36) without the surface free energy (dotted line). Inset: Shapes of a spherical water-rich droplet in equilibrium for $\chi=1.8$, 2.0 , and 2.1 at $v_{0} \bar{n}=6 \times 10^{-4}$, in the region $-400<x, y<400$ and $z=0$, where the colors represent the water composition according to the color bar.

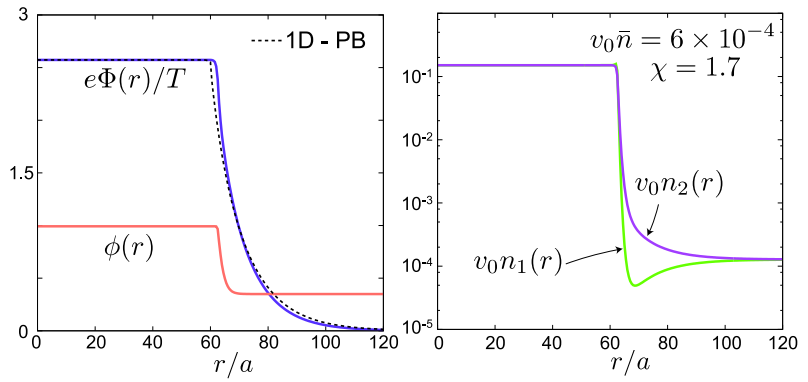

FIG. 12: (Color online) Normalized potential $e \Phi(r) / T$ (with $\left.\Phi_{\beta}=0\right)$ and water volume fraction $\phi(r)$ (left). Normalized ion densities $v_{0} n_{1}(r)$ and $v_{0} n_{2}(r)$ (right). Here $\chi=1.7$ and $e \Delta \Phi=3 T$. The other parameters are the same as in Fig.11. In the phase $\beta$ region near the interface, $\Phi(r)$ well agrees with the one-dimensional solution of the nonlinear PoissonBoltzmann equation (dotted line) and there appears an electric double layer due to nonvanishing $n_{1}-n_{2}$.

\section{B. Proposed experiment: a single droplet}

We performed simulation of a single water-rich droplet in the presence of hydrophilic ions. We have already presented a theory for neutral solute in the same situation in Subsection IIF. Experimentally, wee may also suppose a collection of monodisperse droplets much separated from one another, where the volume per droplet (the inverse of the droplet density) should be treated as the system volume $V$.

The simulation details are as follows. We calculated equilibrium profiles of $\phi(r)$ and $n_{i}(r)$ by minimizing $F$ in Eq.(4.3) around a spherical water-rich droplet with 
radius $R$ in a spherical cell with radius $L=600 a$. At $\bar{\phi}=0.35$, we set (a) $\bar{n}=6 \times 10^{-4}$ or (b) $3 \times 10^{-4}$. The other parameters are $g_{1}=15, g_{2}=7, C=T / a$, and $e^{2} / T=120 a$. The dielectric constant depends on $\phi$ as $\varepsilon=40(1+\phi)$. Since $g_{1}>g_{2}$, an electric double layer is produced at the interface here.

In Fig.11, we show the equilibrium $R$ vs $\chi$ together with shapes of the droplet for three values of $\chi$. The droplet disappears due to the surface tension at $R=$ $18.52 a$ and $\chi=0.799$ for (a) and $R=22.18 a$ and $\chi=$ 1.656 for (b). The corresponding value of $\bar{n} / n_{\mathrm{p}}$ is 1.067 for (a) and 1.102 for (b). These curves are close to those calculated from the approximate free energy (2.36) before the droplet disappearance. The characteristic features can also be calculated from the more approximate free energy (3.14) or (3.20).

In Fig.12, the profiles of the potential $\Phi(r)$ and the ion densities $n_{1}(r)$ and $n_{2}(r)$ are presented for $\chi=1.7$ in the case (a), which follow from Eqs.(3.2) and (3.7). Here $\phi_{\alpha}=0.993$ and $n_{\alpha}=0.352 v_{0}^{-1}$ within the droplet and $\phi_{\beta}=0.349$ and $n_{\beta}=2.55 \times 10^{-4} v_{0}^{-1}$ outside it. The potential $\Phi(r)$ relaxes with the Debye length $\lambda_{\beta}=11.8 a$ and is well fitted to the one-dimensional solution of the nonlinear Poisson-Boltzmann equation (dotted line). In Fig.13, the equilibrium $R$ is displayed as a function of $\chi$ for (a) $v_{0} \bar{n}=6 \times 10^{-4}$ and (b) $3 \times 10^{-4}$. Remarkably, the droplet disappeared at $R=18.52 a$ and $\chi=0.799$ for (a) and at $R=22.18 a$ and $\chi=1.656$ for (b) due to the surface tension effect. This critical radius for (a) nearly coincides with the droplet radius $R=18.23 a$ at the minimum of the curve (b) in Fig.9. We recognize that the relations of $R$ vs $\chi$ here are little affected by the electric double layer. Hence they are close to those of neutral solvent with $g=11$ for the same $\bar{\phi}$ and $\bar{n}$.

\section{HOMOGENEOUS NUCLEATION FROM ONE-PHASE STATES}

We start with homogeneous one-phase states without impurities (other than the solute or the salt under consideration). We assume that the boundary walls are hydrophobic and formation of a water-rich wetting layer is suppressed. In this situation, the precipitation predicted in this paper can be realized via homogeneous nucleation for $\bar{n}>n_{\mathrm{p}}$ or $\chi>\chi_{\mathrm{p}}$. Therefore, we calculate the nucleation rate in the initial stage of nucleation. It is not much affected by the electrostatic interaction for hydrophilic ions and we treat neutral solute in the following. We also neglect temperature inhomogeneity and treat $\chi$ as a homogeneous constant.

It is worth noting that water droplets can easily be produced around hydrophilic ions in metastable gas mixtures containing water vapor. Here ions play the role of nucleation seeds on which hydration-induced condensation is favored. A Ginzburg-Landau approach to this problem was also presented 35 .

\section{A. Linear stability and metastability}

We first examine the linear stability of a one-phase state with $\langle\phi\rangle=\bar{\phi}$ and $\langle n\rangle=\bar{n}$. If $f_{\text {tot }}$ in Eq.(2.3) is expanded with respect to the deviations $\delta \phi=\phi-\bar{\phi}$ and $\delta n=n-\bar{n}$, the second-order free energy deviation is written as

$$
\left(\delta f_{\text {tot }}\right)_{2}=\frac{1}{2} f^{\prime \prime}(\bar{\phi})(\delta \phi)^{2}-T g \delta \phi \delta n+\frac{T}{2 \bar{n}}(\delta n)^{2} .
$$

If the right hand side is nonnegative-definite, the onephase state is stable or metastable. Minimization with respect to $\delta n$ is achieved at $\delta n=g \bar{n} \delta \phi$, leading to $\left(\delta f_{\text {tot }}\right)_{2}=\left[f^{\prime \prime}(\bar{\phi})-T \bar{n} g^{2}\right](\delta \phi)^{2} / 2$. Thus the spinodal curve is expressed as

$$
f^{\prime \prime}(\bar{\phi})-T \bar{n} g^{2}=0 .
$$

See the left panels in Fig.1 for this spinodal curve. Onephase states are linearly stable outside this curve.

In the presence of the Coulomb interaction, the spinodal curve is still given by Eq.(5.2) with the replacement $g=\left(g_{1}+g_{2}\right) / 2$ in Eq.(4.12) under the condition $\gamma_{\mathrm{p}}<1$. Here $\gamma_{\mathrm{p}}$ is a parameter representing the degree of solvation asymmetry between the cations and anions (which should not be confused with the volume fraction $\gamma_{\alpha}$ of phase $\alpha$ ). For monovalent ions it is given by

$$
\gamma_{\mathrm{p}}=(T / 16 \pi C)^{1 / 2}\left|g_{1}-g_{2}\right| .
$$

For most hydrophilic ion pairs, we should have $\gamma_{\mathrm{p}}<1$. On the other hand, the reverse condition $\gamma_{\mathrm{p}}>1$ can be realized for antagonistic salt composed of hydrophilic and hydrophobic ion pairs [13, 22, 23]. Addition of such a salt leads to a decrease in the surface tension and mesophases formation. In this paper, we consider hydrophilic ions with $\gamma_{\mathrm{p}}<1$ for simplicity.

\section{B. Droplet free energy and nucleation rate}

In the early stage of nucleation, the volume fraction $\gamma_{\alpha}$ of the new phase is very small and the droplets may be treated independently. Hence we consider a single droplet with radius $R$ in the classical nucleation theory 32]. The composition and the solute density far from the droplet are $\bar{\phi}$ and $\bar{n}$. A key quantity here is the free energy $\Delta F(R)$ needed to create a droplet of the new phase.

To derive $\Delta F(R)$, we use the total free energy density $f_{\text {tot }}(\phi, n)$ in Eq.(2.3). It is equal to $\bar{f}_{\text {tot }}=f_{\text {tot }}(\bar{\phi}, \bar{n})$ in the initial homogeneous state and remains so far from the droplet, while it is equal to $f_{\text {tot }}^{\alpha}=f_{\text {tot }}\left(\phi_{\alpha}, n_{\alpha}\right)$ inside the droplet. Here $\phi_{\alpha}$ and $n_{\alpha}$ are the composition and the solute density inside the droplet, respectively. The change of the total free energy is

$$
\begin{aligned}
& \Delta F(R)=\frac{4 \pi}{3} R^{3}\left[f_{\text {tot }}^{\alpha}-\bar{f}_{\text {tot }}\right] \\
& +\int_{r>R} d \boldsymbol{r}\left[f_{\text {tot }}(\phi, n)-\bar{f}_{\text {tot }}\right]+4 \pi \sigma R^{2} .
\end{aligned}
$$


In the right hand side, the first term is the contribution from the droplet interior, the second term is that of the droplet exterior, and the last term is the surface tension term. Outside the droplet we assume small deviations of $\phi$ and $n$ from the initial values $\bar{\phi}$ and $\bar{n}$ so that we set $f_{\text {tot }}(\phi, n)-\bar{f}_{\text {tot }}=\bar{h}(\phi-\bar{\phi})+\bar{\mu}(n-\bar{n})$, where $\bar{h}$ and $\bar{\mu}$ are the values of $h$ in Eq.(2.6) and $\mu$ in Eq.(2.7) in the initial metastable state. Further we note that the integrals of $\phi-\bar{\phi}$ and $n-\bar{n}$ in the whole space vanish from the conservation relations $(2.5)$, so that their space integrals outside the droplet are equal to $-(4 \pi / 3) R^{3}\left(\phi_{\alpha}-\bar{\phi}\right)$ and $-(4 \pi / 3) R^{3}\left(n_{\alpha}-\bar{n}\right)$, respectively. Therefore, it follows the standard form [32],

$$
\Delta F(R)=-\frac{4 \pi}{3} R^{3} w+4 \pi R^{2} \sigma .
$$

The coefficient $w$ represents the degree of metastability and is of the form,

$$
\begin{aligned}
-w & =f_{\mathrm{tot}}^{\alpha}-\bar{f}_{\mathrm{tot}}-\bar{h}\left(\phi_{\alpha}-\bar{\phi}\right)-\bar{\mu}\left(n_{\alpha}-\bar{n}\right) \\
& =\Omega\left(\phi_{\alpha}\right)-T \bar{n} \Psi\left(\phi_{\alpha}\right),
\end{aligned}
$$

where $\Omega\left(\phi_{\alpha}\right)$ and $\Psi\left(\phi_{\alpha}\right)$ are given in Eqs.(2.37) and (2.38). From the second line of Eq.(5.6), this $w$ coincides with $w$ introduced in Eq.(3.15). It is proportional to $\bar{n}-n_{\mathrm{p}}$ and $\chi-\chi_{\mathrm{p}}$ as in Eqs.(3.16) and (3.17), where $n_{\mathrm{p}}$ and $\chi_{\mathrm{p}}$ are defined in Eqs.(2.46) and (2.48).

The critical radius is $R_{c}=2 \sigma / w$ as in Eq.(3.18). At $R=R_{c}, \Delta F(R)$ attains a maximum given by

$$
F_{c}=16 \pi \sigma^{3} / 3 w^{2}
$$

In the classical nucleation theory, critical droplets appear with a constant rate $I$ per unit time and per unit volume. It is called the nucleation rate and is of the form,

$$
I=I_{0} \exp \left(-F_{c} / T\right),
$$

where the coefficient $I_{0}$ depends on the dynamics and will be estimated in Eq.(5.22). For weal metastability, the nucleation barrier $F_{c} / T$ grows as $w^{-2}$ and $I$ is very sensitive to $w$. From Eqs.(5.7) and (5.8) the nucleation barrier is expressed as

$$
\begin{aligned}
F_{c} / T & =A_{c} /\left(\bar{n} / n_{\mathrm{p}}-1\right)^{2} \\
& =B_{c} /\left(\chi-\chi_{\mathrm{p}}\right)^{2} .
\end{aligned}
$$

The two coefficients $A_{c}$ and $B_{c}$ are defined by

$$
\begin{aligned}
& A_{c}=16 \pi \sigma^{3} / 3 \Omega^{2}, \\
& B_{c}=16 \pi \sigma^{3} v_{0}^{2} / 3 T^{2}\left(\phi_{\alpha}-\bar{\phi}\right)^{4},
\end{aligned}
$$

where $A_{c}$ depends on $\chi$ and $\bar{\phi}$ and $B_{c}$ on $\bar{n}$ and $\bar{\phi}$. We may use Eq.(5.9) or Eq.(5.10) depending on whether $\bar{n}$ or $\chi=\chi(T)$ is varied.

In Fig.13, we plot $R_{c}$ vs $\bar{n} / n_{\mathrm{p}}-1$ at fixed $\chi$ in the left and $R_{c}$ vs $\chi-\chi_{\mathrm{p}}$ at fixed $\bar{n}$ in the right. It much exceeds the molecular size $a$ for weak metastability, where $\bar{n} / n_{\mathrm{p}}-$ 1 or $\chi-\chi_{\mathrm{p}}$ is small. In Fig.14, we plot $A_{c}$ at fixed $\chi$
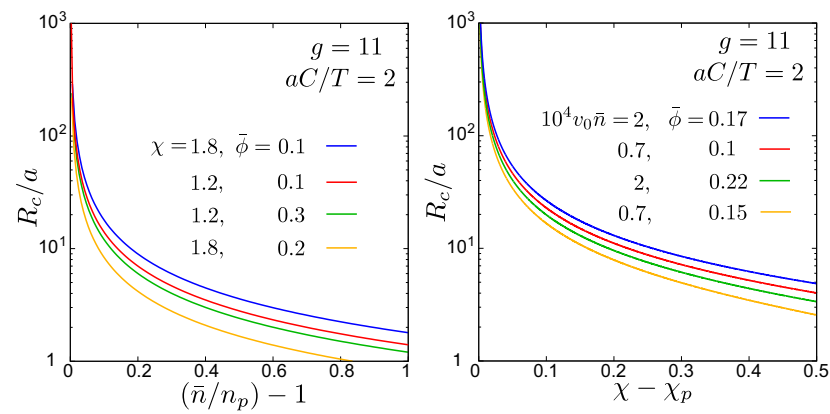

FIG. 13: (Color online) Critical radius $R_{c}$ in Eq.(3.18) as a function of $\bar{n} / n_{\mathrm{p}}-1$ for $(\chi, \bar{\phi})=(1.8,0.1),(1.2,0.1),(1.2,0.3)$, and $(1.8,0.2)$ from above (left) and as a function of $\chi-\chi_{\mathrm{p}}$ for $\left(10^{4} v_{0} \bar{n}, \bar{\phi}\right)=(2.0 .17),(0.7,0.1),(2,0.22)$, and $(0.7,0.15)$ from above (right). Here $g=11$ and $C=2 T / a$.
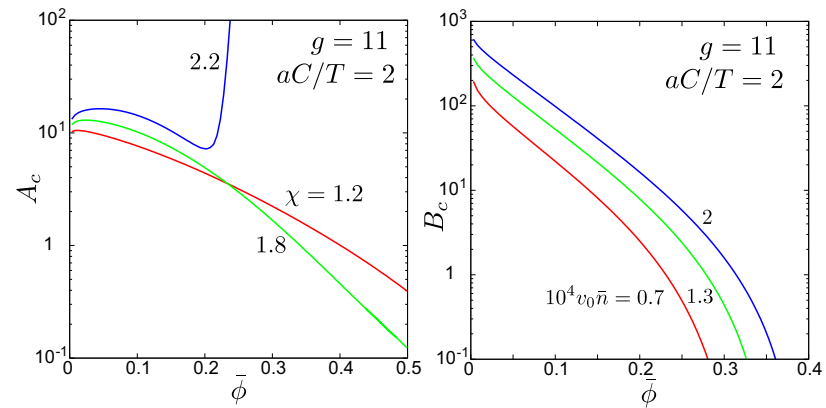

FIG. 14: (Color online) $A_{c}$ at fixed $\chi$ in Eq.(5.11) (left) and $B_{c}$ at fixed $\bar{n}$ in Eq.(5.12) (right) as functions of $\bar{\phi}$ for $g=11$ and $C=2 T / a$. For $\chi>2$ (left), there is no barrier or $A_{c} \rightarrow \infty$ as $\bar{\phi}$ approaches the equilibrium coexistence composition.

and $B_{c}$ at fixed $\bar{n}$ as functions of $\bar{\phi}$ for $g=11$. These coefficients are considerably larger than unity for $\bar{\phi} \lesssim 0.2$, but become small with increasing $\bar{\phi}$. In these figures we set $C=2 T / a$. Here notice the relations $R_{c} \propto C^{1 / 2}$, $\sigma \propto C^{1 / 2}$, and $F_{c} \propto C^{3 / 2}$. The coefficients $A_{c}$ and $B_{c}$ in Eqs. (5.11) and (5.12) depend on $C$ as $\sigma^{3} \propto \xi^{3} \propto C^{3 / 2}$. For aqueous fluids with the hydrogen bonding network, a larger value of $C$ might be more appropriate than in Figs.13 and 14. See the summary for more discussions on the choice of $C$.

Nucleation experiments have been performed precisely on near-critical binary mixtures (without salt) [32, 3840], where appreciable droplets become observable for $F_{c} / T \lesssim 50$. From Fig. 14, we find $F_{c} / T \lesssim 50$ for $\bar{n} / n_{\mathrm{p}} \gtrsim 1.2$ at $\bar{\phi}=0.1$ In our case, once $\bar{n}>n_{\mathrm{p}}$ or $\chi>\chi_{\mathrm{p}}$, the nucleation rate should soon becomes large enough for usual observations of droplets with $R>R_{c}$.

\section{Weak metastability and droplet growth}

We may derive an approximate expression for $w$ in terms of $\bar{\phi}$ and $\bar{n}$ for weak metastablilty. To this end, we 
consider an equilibrium reference state, where two bulk phases with $(\phi, n)=\left(\phi_{\alpha}, n_{\alpha}\right)$ and $\left(\phi_{\beta}, n_{\beta}\right)$ are separated by a planar interface. We assume that the composition and the solute density $\left(\phi_{\alpha}, n_{\alpha}\right)$ in the reference phase $\alpha$ are the same as those within the droplet. However, those $\left(\phi_{\beta}, n_{\beta}\right)$ in the reference phase $\beta$ are slightly different from those $(\bar{\phi}, \bar{n})$ in the initial metastable state. The initial homogeneous deviations are written as

$$
\delta \bar{\phi}=\bar{\phi}-\phi_{\beta}, \quad \delta \bar{n}=\bar{n}-n_{\beta} .
$$

From Eqs.(2.16) and (2.17) we obtain

$$
f_{\mathrm{tot}}^{\alpha}-f_{\mathrm{tot}}^{\beta}-h_{\mathrm{cx}} \Delta \phi-\mu_{\mathrm{cx}} \Delta n=0,
$$

where $f_{\text {tot }}^{K}=f_{\text {tot }}\left(\phi_{K}, n_{K}\right)($ with $\left.K=\alpha, \beta)\right)$. The $h_{\mathrm{cx}}$ and $n_{\mathrm{cx}}$ are the values of $h$ in Eq.(2.6) and $\mu$ in Eq.(2.7) in the reference state (which are simply written as $h$ and $\mu$ in Sec.II). Further we note the relation,

$$
\bar{f}_{\text {tot }}-f_{\text {tot }}^{\beta}-h_{\mathrm{cx}} \delta \bar{\phi}-\mu_{\mathrm{cx}} \delta \bar{n} \cong 0,
$$

which is valid to first order in $\delta \bar{\phi}$ and $\delta \bar{n}$. From the first line of Eq.(5.6) we may eliminate $f_{\text {tot }}^{K}$ and $\bar{f}_{\text {tot }}$ to obtain the desired expression,

$$
w \cong\left(\bar{h}-h_{\mathrm{cx}}\right) \Delta \phi+\left(\bar{\mu}-\mu_{\mathrm{cx}}\right) \Delta n,
$$

where we have set $\phi_{\alpha}-\bar{\phi} \cong \Delta \phi$ and $n_{\alpha}-\bar{n} \cong \Delta n$. From Eqs.(2.6) and (2.7) the initial deviations of the chemical potentials are expanded as

$$
\begin{aligned}
\bar{h}-h_{\mathrm{cx}} & \cong f_{\beta}^{\prime \prime} \delta \bar{\phi}-T g \delta \bar{n} \\
\bar{\mu}-\mu_{\mathrm{cx}} & \cong T \delta \bar{n} / n_{\beta}-T g \delta \bar{\phi},
\end{aligned}
$$

where $f_{\beta}^{\prime \prime}$ is equal to $\partial^{2} f / \partial \phi^{2}$ at $\phi=\phi_{\beta}$. Therefore,

$$
\frac{w}{T} \cong\left(f_{\beta}^{\prime \prime} \frac{\Delta \phi}{T}-g \Delta n\right) \delta \bar{\phi}+\left(e^{g \Delta \phi}-1-g \Delta \phi\right) \delta \bar{n} .
$$

In Appendix B, we will derive the dynamic equation for the droplet radius $R(t)$ for weak metastability. It is of the standard form,

$$
\frac{\partial R}{\partial t}=\frac{\Lambda}{R}\left(w-\frac{2 \sigma}{R}\right),
$$

where $\Lambda$ is a kinetic coefficient defined in Eq.(B10) below. For dilute solute, it may be related to the mutual diffusion constant of the mixture $D_{m}(\phi)$ by

$$
\Lambda=D_{m}(\bar{\phi}) / f^{\prime \prime}(\bar{\phi})(\Delta \phi)^{2} .
$$

For $R \cong R_{c}$ we have $\partial R / \partial t \cong \Gamma_{c}\left(R-R_{c}\right)$, where

$$
\Gamma_{c}=2 \sigma \Lambda / R_{c}^{3} .
$$

The inverse $\Gamma_{c}^{-1}$ is the time scale of near-critical droplets. The coefficient $I_{0}$ in the nucleation rate $(5.8)$ is determined by the droplet dynamics at $R \cong R_{c}$ (with appropriate thermal noises added) and is estimated as [32]

$$
I_{0} \sim \Gamma_{c} / \xi^{3}
$$

where $\xi$ is the correlation length in the initial metastable state.

For $g=0$ our results tend to those for incompressible binary mixtures without salt [32, 38, 39]. There, we use Eq.(5.22) and the relation $w-2 \sigma / R=f_{\beta}^{\prime \prime}(\Delta \phi)^{2}\left[\Delta_{s}-\right.$ $\left.2 d_{0} / R\right]$ in terms of the supersaturation $\Delta_{s}=\delta \bar{\phi} / \Delta \phi$ and the capillary length $d_{0}=\gamma / f_{\beta}^{\prime \prime}(\Delta \phi)^{2}$.

\section{HETEROGENEOUS NUCLEATION ON HYDROPHILIC COLLOID SURFACES}

In this section, we examine adsorption, wetting, and precipitation on a colloid surface. Here the colloid surfaces are hydrophilic, while the boundary walls are hydrophobic as in the previous section. The colloid density $n_{\text {co }}$ is so small that each colloid particle may be treated independently and the composition profile around it depends only on the distance $r$ from its center. The effective cell volume for each particle is $V=n_{\text {co }}^{-1}$. For simplicity, we assume that the colloids are neutral without surface charge and the correlation length $\xi$ outside the colloids is shorter than the colloid radius $d$.

In Sec.IIIC, we have already found a prewetting transition on a planar wall for $\chi$ slightly below $\chi_{\mathrm{p}}$. Furthermore, we shall see that a wetting layer much thickens slightly above $\chi_{\mathrm{p}}$ due to precipitation.

\section{A. Simulation results: discontinuous and continuous transitions}

First, we present our numerical results for a hydrophilic particle with radius $d=15 a$ or $25 a$ placed at the center of a spherical cell with radius $L=10^{3} \mathrm{a}$. The cell volume is $V=4 \pi L^{3} / 3$ and the colloid volume fraction is $\phi_{\text {col }}=(d / L)^{3}$, so $\phi_{\text {col }}=3.4 \times 10^{-6}$ and $1.6 \times 10^{-5}$ for $d=15 a$ and $25 a$, respectively. We suppose hydrophilic ions using the model in Sec.IV, though the electrostatic interaction is not essential here. The other parameters used are $\bar{\phi}=0.36, v_{0} n_{0}=2 \times 10^{-4}, g_{1}=9, g_{2}=13$, $C=T / a, e^{2} / T=120 a$, and $\varepsilon_{1}=\varepsilon_{0}=40$. The correlation length $\xi$ far from the surface is shorter than $d$. In fact, it is $1.39 a$ at $\chi=1.9$.

For the composition $\phi$ and the ion densities $n_{1}$ and $n_{2}$, the total free energy is $F_{\text {tot }}=F+F_{s}$, where $F$ is given by Eq.(4.3) and $F_{s}$ is the surface free energy in Eq.(3.27). The composition $\phi(r)$ obeys Eq.(3.2) at a constant $h$. The boundary conditions at $r=d$ and $L$ are given by

$$
C \phi^{\prime}(d)=-\alpha_{w}, \quad \phi^{\prime}(L)=0,
$$

where $\phi^{\prime}(r)=d \phi(r) / d r$. The wetting parameter $\alpha_{\mathrm{w}}$ is set equal to $0.165 T / a^{2}$ as in Fig. 10.

In Fig.15, we plot the preferential adsorption given by

$$
\Gamma=\int_{r>d} d \boldsymbol{r}[\phi(r)-\phi(L)],
$$




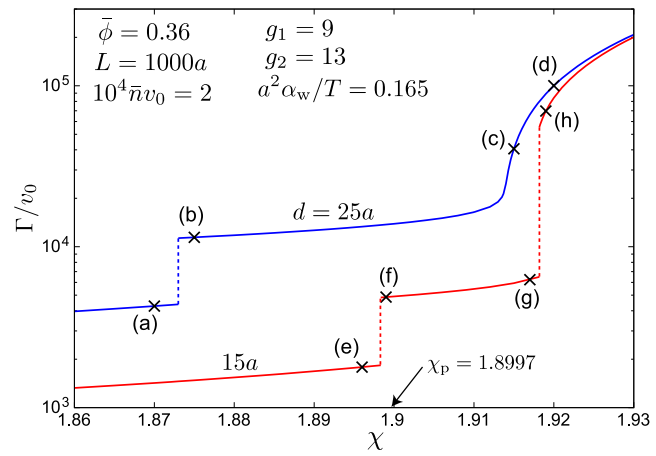

FIG. 15: Preferential adsorption $\Gamma$ on a colloid surface in Eq.(6.2) as a function of $\chi$ on a semi-logarithmic scale at $\bar{\phi}=$ 0.36 . For $d=25 a$ (upper curve), after a prewetting transition at $\chi=1.873$, the crossover to precipitation is continuous. For $d=15 a$ (lower curve), after a prewetting transion at $\chi=$ 1.898, the transition to precipitation is discontinuous at $\chi=$ 1.918. At the points (a)-(h), the corresponding composition profiles are given in Fig.16.

for the two diameters $d=25 a$ and $15 a$. The prewetting transition discussed in Sec.IIIC occurs at $\chi=1.873$ for $d=25 a$ and 1.893 for $d=15 a$. Remarkably, for $\chi>\chi_{\mathrm{p}}=1.8997, \Gamma$ increases continuously for $d=25 a$, but discontinuously for $d=15 a$. In Fig.16, we show the composition $\phi(r)$ for four $\chi$ across the transitions and around the crossover. It changes from a thin to thick wetting layer continuously (but abruptly) for $d=25 a$ and discontinuously for $d=15 a$. In Fig.17, we show the free energy change $\Delta F_{\text {tot }}=F_{\text {tot }}-F_{\text {tot }}^{0}$ as a function of $\chi$, where $F_{\text {tot }}^{0}$ is the total free energy for the homogeneous state (realized for $\alpha_{w}=0$ ). Around the first-order transitions there appear two branches, where Eq.(3.2) is satisfied, and on the equilibrium branch $F_{\text {tot }}$ takes a lower value. In particular, for $d=15 a$, two branches of adsorption and precipitation appear around $\chi=1.918>\chi_{\mathrm{p}}$.

The prewetting transition point below $\chi_{\mathrm{p}}$ depends on $d$ as in Fig.15. Furthermore, it is slightly affected by the electric double layer for $g_{1} \neq n_{2}$. In fact, for $g_{1}=g_{2}=$ 11 , it disapperas and the prewetting transition occurs at $\chi=1.907$ for $d=25 a$ and at $\chi=1.912$ for $d=15 a$, while it was at $\chi=1.885$ for a planar wall in Fig.10.

\section{B. Theory of wetting on a colloid surface}

Before the layer thickening due to precipitation and for $d \gg \xi, \Delta F_{\text {tot }}$ is due to the adsorption expressed in terms of $\mathcal{F}_{\text {ad }}$ in Eq.(3.29) as

$$
\Delta F_{\text {tot }}=4 \pi d^{2} \mathcal{F}_{\text {ad }}(\bar{\phi}, \bar{n}),
$$

We explicitly write the dependence of $\mathcal{F}_{\text {ad }}$ on the composition $\bar{\phi}$ and the solute density $\bar{n}$ far from the surface. In Fig.17, $\mathcal{F}_{\text {ad }}$ only weaky depends on $\chi$. After precipitation in the range $\chi>\chi_{\mathrm{p}}$, a thick wetting layer appears
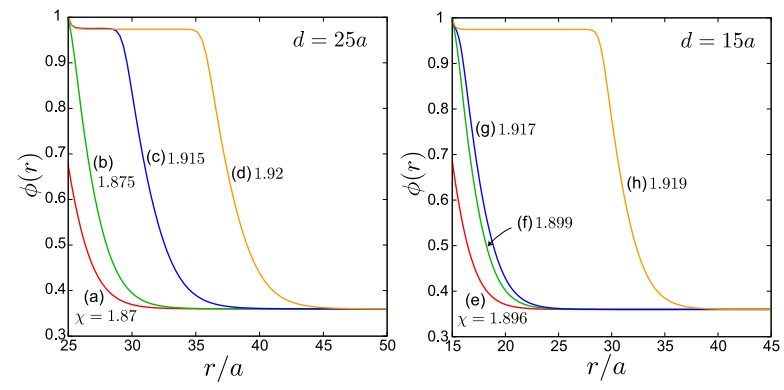

FIG. 16: (Color online) Composition $\phi(r)$ vs $r / a$ outside a colloid $r>d$. For $d=25 a$ (left), $\chi$ is (a) 1.87, (b) 1.875 , (c) 1.915 , and (d) 1.92. For $d=15 a$ (right), $\chi$ is (e) 1.896, (f) $1.899,(\mathrm{~g}) 1.917$, and (h) 1.919 .
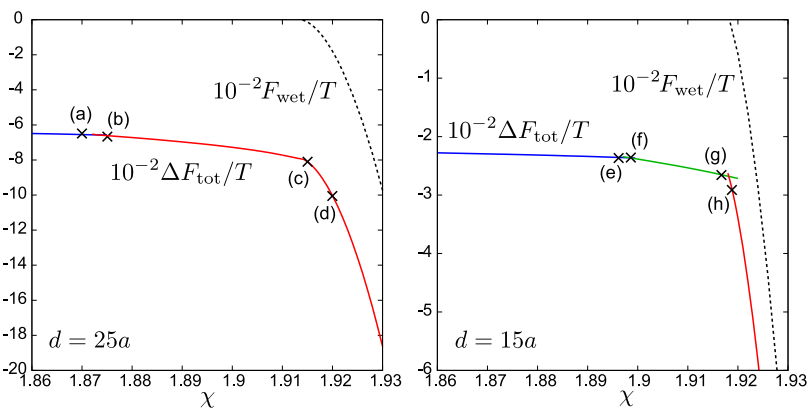

FIG. 17: (Color online) Free energy change $\Delta F_{\text {tot }}$ due to water accumulation around a colloid as a function of $\chi$ for $\bar{\phi}=$ 0.36 . For $d=25 a$ (left), after the prewetting transion between (a) and (b), the crossover to precipitation is continuous but there is an abrupt slope change at (c). For $d=15 a$ (right) after the prewetting transion between (e) and (f), there is a discontinuous change between $(\mathrm{g})$ and $(\mathrm{h})$. Shown also is the approximate free energy $F_{\text {wet }}$ in Eq.(6.6) (dotted line).

in the region $d<r<R$ and $\Delta F_{\text {tot }}$ is expressed as

$$
\Delta F_{\text {tot }}=4 \pi d^{2} \mathcal{F}_{\text {ad }}\left(\phi_{\alpha}, n_{\alpha}\right)+F_{\text {wet }} .
$$

The first adsorption term is determined by the composition $\phi_{\alpha}$ and the solute density $n_{\alpha}$ in the surrounding thick layer, while the second one is the contribution in the thick layer. Figure 17 indicates that $F_{\text {wet }}$ decreases dramatically with increasing $\chi$.

To construct a simple theory of $F_{\text {wet }}$, we assume the condition $\Psi\left(\phi_{\alpha}\right) \gamma_{\alpha} \ll 1$ in Eq.(3.13). We treat $\Delta F_{\text {tot }}$ in Eq.(2.65) as $F_{\text {wet }}$. In the present situation, the volume fraction of phase $\alpha$ is given by

$$
\gamma_{\alpha}=4 \pi\left(R^{3}-d^{3}\right) / 3 V=\left(R^{3}-d^{3}\right) / L^{3} .
$$

Here $w$ in Eq.(3.15) is positive. In terms of the two lengths $R_{c} \propto w^{-1}$ in Eq.(3.18) and $R_{\mathrm{m}}$ in Eq.(3.19), we may express $F_{\text {wet }}$ as

$$
\begin{aligned}
\frac{F_{\mathrm{wet}}}{4 \pi \sigma} & =R^{2}-d^{2}-\frac{2}{3 R_{c}}\left(R^{3}-d^{3}\right)+\frac{1}{3 R_{\mathrm{m}}^{4}}\left(R^{3}-d^{3}\right)^{2} \\
& =R_{\mathrm{m}}^{2}\left[\left(q+D^{3}\right)^{2 / 3}-D^{2}-\frac{2 E}{3} q+\frac{1}{3} q^{2}\right] . \quad(6.6
\end{aligned}
$$


The first line follows from Eq.(3.20) if $R^{2}$ and $R^{3}$ there are replaced by $R^{2}-d^{2}$ and $R^{3}-d^{3}$, respectively. Thus, as $d \rightarrow 0$, this $F_{\text {wet }}$ tends to $\Delta F_{\text {tot }}$ in Eq.(3.20). In the second line we introduce the order parameter,

$$
q=\left(R^{3}-d^{3}\right) / R_{\mathrm{m}}^{3},
$$

where $D$ and $E$ are dimensionless parameters defined as

$$
D=d / R_{\mathrm{m}}, \quad E=R_{\mathrm{m}} / R_{c}=w R_{\mathrm{m}} / 2 \sigma .
$$

We minimize $F_{\text {wet }}$ as a function of $R$ or $q$. In Fig.17, this $F_{\text {wet }}$ is plotted (in dotted lines) and is compared with $\Delta F_{\text {tot }}$. In Fig.18, we display $q$ in the $D-E$ plane, which shows how a thick wetting layer appears. For $D=0$ (or $d=0$ ) we have the curve of $R^{3} / R_{\mathrm{m}}^{3}$ vs $R_{\mathrm{m}} / R_{c}$ for a droplet with radius $R$ (see Sec.IIF for its theory).

For $d-R \gg \xi$, the adsorption free energy $4 \pi d^{2} \mathcal{F}_{\text {ad }}$ is small as compared to $F_{\text {wet }}$. Then we may examine the formation of a thick layer by minimizing $F_{\text {wet }}$. For $q \ll D^{3}, F_{\text {wet }}$ is expanded in powers of $q$ as

$$
\frac{F_{\mathrm{wet}}}{4 \pi \sigma R_{\mathrm{m}}^{2}}=\frac{2}{3}\left(\frac{1}{D}-E\right) q+\left(3-\frac{1}{D^{4}}\right) \frac{q^{2}}{9}+\frac{4 q^{3}}{81 D^{7}}+\cdots
$$

By setting the coefficient of $q^{2}$ equal to zero, we find a tricritical value of $D$ given by

$$
D_{\text {tri }}=3^{-1 / 4}=0.760 .
$$

For $D=d / R_{\mathrm{m}}<D_{\text {tri }}$, the second term $\left(\propto q^{2}\right)$ in the expansion (6.9) is negative and the transition takes place discontinuously on the transition line $E=E_{\mathrm{tr}}(D)$, where $E_{\text {tr }} \rightarrow D_{\text {tri }}^{-1}$ as $D \rightarrow D_{\text {tri }}$ and $E_{\text {tr }} \rightarrow 2$ as $D \rightarrow 0$. On the other hand, for $D=d / R_{\mathrm{m}}>D_{\text {tri }}$, a thick wetting layer appears continuously as a second-order phase transition. The parameter $q$ is nonvanishing for

$$
E>D^{-1} \quad \text { or } \quad d>R_{c}
$$

where $q \propto\left(E-D^{-1}\right)^{1 / 2}$. Thus a tricritical point is at $(D, E)=\left(D_{\text {tri }}, D_{\text {tri }}^{-1}\right)$ or at $(d, w)=\left(D_{\text {tri }} R_{\mathrm{m}}, 2 \sigma / D_{\text {tri }} R_{\mathrm{m}}\right)$ for precipitation on a colloid surface. In Figs.15-17, we have $R_{\mathrm{m}}=26 a$, so that $D=0.96>D_{\text {tri }}$ for $d=25 a$ and $D=0.58<D_{\text {tri }}$ for $d=15 a$. Our numerical results are consistent with the prediction from Eq.(6.6).

\section{SUMMARY AND REMARKS}

In summary, we have examined solvation-induced precipitation in aqueous mixtures with hydrophilic or hydrophobic solute in the limit of large $g$, which represents the composition-dependence of the solvation (the strength of the preferential solvation).

In Sec.II, we have developed a thermodynamic theory of two-phase coexistence in mixture solvents with solute, numerical analysis of the two-phase coexistence, a theory of precipitation in the asymptotic limit $g \gg 1$, and

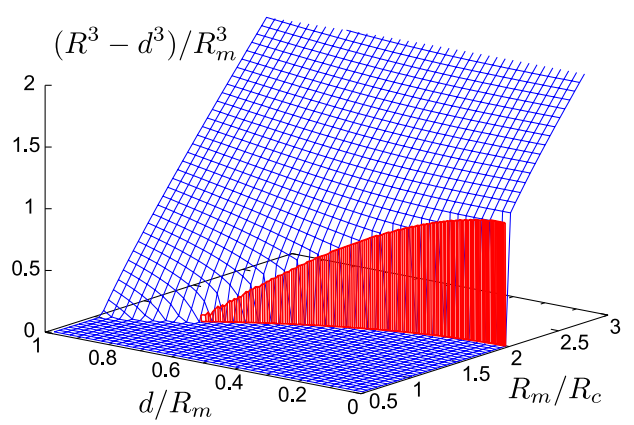

FIG. 18: (Color online) Precipitated layer volume $4 \pi\left(R^{3}-\right.$ $\left.d^{3}\right) / 3$ divided by $4 \pi R_{\mathrm{m}}^{3} / 3$ in the plane of $d / R_{\mathrm{m}}$ and $R_{\mathrm{m}} / R_{c}$. It is calculated from the approximate wetting free energy $F_{\text {wet }}$ in Eq.(6.6) without the adsorption free energy $4 \pi d^{2} \mathcal{F}_{\text {ad }}$. The transition is discontinuous for $d / R_{\mathrm{m}}<0.760$ on the perpendicular surface (in red).

a theory in the dilute limit of precipitated domains. Remarkably, the precipitation curve $\chi=\chi_{\mathrm{p}}$ extends far below the coexistence curve in the $\chi-\bar{\phi}$ plane. In Sec.III, introducing the gradient free energy, we have calculated the surface tension $\sigma$, performed stability analysis of a precipitated droplet including the surface tension, and predicted a prewetting transition below the precipitation curve. In Sec.IV, we have presented a theory for hydrophilic ions including the solvation and electrostatic interactions. Finally, we have investigated homogeneous nucleation in Sec.V and heterogeneous nucleation on a collod surface in Sec.VI. We have also derived the evolution equation for the droplet radius $R(t)$ from the dynamic equations for $\phi$ and $n$ in metastable states in Appendix B.

We have presented a number of predictions. They are (i) droplet appearance due to preferential solvation as in Figs.1-3, which has a minimim size $R_{\mathrm{m}}$ in Eq.(3.19), (ii) droplet size change with varying $\chi$ or $T$ as in Fig.11, (iii) prewetting transition slightly below the precipitation curve, (iv) nucleation slightly above the precipitation curve and far outside the solvent coexistence curve, and (v) heterogeneous nucleation on a collod surface, where two-step first-order wetting transitions can occur as in Figs.15-18. To confirm these effects, systematic experiments are needed, where the temperature, the water volume fraction, and the salt amount are controlled. Particularly, dynamic light scattering is informative to detect emergence of droplets and wetting on colloid surfaces. Wetted colloids should move with a smaller diffusion constant $\propto R^{-1}$.

In our theory, the molecular volumes of the two components are assumed to be given by the common $v_{0}$ in Eq.(2.4). However, they can be very different. For example, those of $\mathrm{D}_{2} \mathrm{O}$ and $3 \mathrm{MP}$ (the inverse densities of the pure components) are 28 and $168 \AA^{3}$, respectively. Moreover, the coefficient $C$ in Eq.(3.1) of the gradient free energy remains an arbitrary constant and the nucleation rate calculated in Sec. $\mathrm{V}$ is proportional to $C^{3 / 2}$. 
Therefore, our theory is still very qualitative. For pure water, the observed surface tension outside the critical region $1-T / T_{c} \gtrsim 0.1$ fairly agrees with the calculated surface tension from the van der Waals model with the gradient free energy density $10 T a^{5}|\nabla n|^{2} / 2[35,41]$, where $n$ is the density and $a=3.1 \AA$ is the van der Waals radius.

In future, we will investigate the wetting transition on charged walls, rods, and colloids and the solvationinduced colloid interaction, which can be much influenced by the ion-induced precipitation. The degree of ionization should also be treated as a fluctuating variable sensitively depending on the local environment [29].

\section{Acknowledgments}

One of the authors (A.O.) would like to thank M.A. Anisimov for valuable discussions in an early stage of this work. This work was supported by Grant-in-Aid for Scientific Research on Priority Area "Soft Matter Physics" from the Ministry of Education, Culture, Sports, Science and Technology of Japan.

\section{Appendix A: Two-phase coexistence for $\chi>2$ at very small $\bar{n}$}

For $\chi>2$ two-phase coexistence is possible without solute. Let $\phi_{\alpha}^{0}$ and $\phi_{\beta}^{0}$ be the equilibrium volume fractions in the two phases without solute. Here we calculate the deviations of the volume fractions $\delta \phi_{\alpha}=\phi_{\alpha}-\phi_{\alpha}^{0}$ and $\delta \phi_{\beta}=\phi_{\beta}-\phi_{\beta}^{0}$ to linear order in the solute density $\bar{n}$ in the mean field theory. We show that the linear theory holds only for very small $\bar{n}$.

The $n_{\alpha}$ and $n_{\beta}$ are still given by Eqs.(2.8) and (2.9). From Eqs.(2.15) and (2.17) the chemical potential difference $h$ is expanded as $h=-T \Delta n / \Delta \phi$ so that

$$
\begin{aligned}
& \delta \phi_{\alpha}=T n_{\alpha}\left(g \Delta \phi-1+e^{-g \Delta \phi}\right) / f^{\prime \prime} \Delta \phi, \\
& \delta \phi_{\beta}=T n_{\beta}\left(g \Delta \phi+1-e^{g \Delta \phi}\right) / f^{\prime \prime} \Delta \phi,
\end{aligned}
$$

where $f^{\prime \prime}=f^{\prime \prime}\left(\phi_{\alpha}^{0}\right)=f^{\prime \prime}\left(\phi_{\beta}^{0}\right)$ for the symmetric free energy density in Eq.(2.4). Here $f^{\prime \prime}(\phi)=\partial^{2} f / \partial \phi^{2}$.

As the solvent critical point $\left(\chi_{c}=2\right)$ is approached, the inequality $\Delta \phi \ll 1 / g$ holds eventually to give

$$
\delta \phi_{\alpha} \cong-\delta \phi_{\beta} \cong T \bar{n} g^{2} \Delta \phi / 2 f^{\prime \prime} .
$$

If we set $\epsilon=\chi-2\left(\propto 1-T / T_{c}\right)$, the above deviation is of order $v_{0} \bar{n} \epsilon^{-1 / 2}$. The linear approximation holds when $\delta \phi_{\alpha} \ll \phi_{\alpha}^{0}-1 / 2 \cong \Delta \phi / 2$. Thus the upper bound of the linear regime of the solute doping is very small as

$$
\bar{n} \ll \epsilon^{3 / 2} / g^{2} v_{0} .
$$

In the left panels of Fig.1, $\phi_{\alpha}-\phi_{\beta}$ is not small even for $\chi \cong 2$ due to the nonlinear solute effect.

Away from the critical point, the relation $g \Delta \phi \gg 1$ holds for large $g$. In this case, we find

$$
\delta \phi_{\alpha} \cong T g n_{\alpha} / f^{\prime \prime}, \quad \delta \phi_{\beta} \cong-T n_{\alpha} / f^{\prime \prime} \Delta \phi,
$$

so that $\delta \phi_{\alpha} \cong-(g \Delta \phi) \delta \phi_{\beta}>0$. From Eq. (2.13) $\delta \phi_{\alpha}(\propto$ $n_{\alpha}$ ) increases steeply as $\bar{\phi}$ is decreased to $\phi_{\beta}^{0}$ (or as $\gamma_{\alpha}$ is decreased). This tendency can be seen in the left panels of Fig.1. The maximum of $n_{\alpha}$ is $\bar{n} e^{g \Delta \phi}$. The condition that $\delta \phi_{\alpha}$ is much smaller than unity is written as

$$
\bar{n} \ll f^{\prime \prime} e^{-g \Delta \phi} / T g
$$

If Eq.(A3) or Eq.(A5) does not hold, $\phi_{\alpha}$ nonlinearly deviates from $\phi_{\alpha}^{0}$ with respect to $\bar{n}$.

\section{Appendix B: Droplet growth}

For weak metastability, we derive the droplet-evolution equation (5.19) in the neutral solute case. The simplest dynamic equations for the composition $\phi(\boldsymbol{r}, t)$ and the solute density $n(\boldsymbol{r}, t)$ are given by the diffusive equations,

$$
\begin{aligned}
& \frac{\partial \phi}{\partial t}=L \nabla^{2}\left(h-C \nabla^{2} \phi\right), \\
& \frac{\partial n}{\partial t}=D_{s} \nabla \cdot n \nabla \cdot(\mu / T),
\end{aligned}
$$

where $L$ is the kinetic coefficient for the composition, $D_{s}$ is the solute diffusion constant, and $h$ and $\mu$ are given in Eqs.(2.6) and (2.7). Around a spherical droplet with radius $R$, all the quantities depend on the distance $r$ from the droplet center and the time $t$.

Slightly outside the droplet surface $r-R \gtrsim \xi$, the gradient term in $h$ is negligible, where $\xi$ is the interface thickness. When the droplet growth or shrinkage is slow, we may use the quasi-static approximation:

$$
\begin{aligned}
& h \cong \bar{h}+\left(h_{R}-\bar{h}\right) R / r, \\
& \mu \cong \bar{\mu}+\left(\mu_{R}-\bar{\mu}\right) R / r,
\end{aligned}
$$

where $h$ and $\mu$ tend to $\bar{h}$ and $\bar{\mu}$ far from the droplet and to $h_{R}$ and $\mu_{R}$ near the droplet surface. The conservations of $\phi$ and $n$ at the interface yield

$$
\begin{aligned}
& \frac{\partial R}{\partial t} \Delta \phi=\frac{L}{R}\left(h_{R}-\bar{h}\right), \\
& \frac{\partial R}{\partial t} \Delta n=\bar{n} \frac{D_{s}}{R}\left(\mu_{R}-\bar{\mu}\right),
\end{aligned}
$$

where $\Delta \phi=\phi_{\alpha}-\phi_{\beta}$ and $\Delta n=n_{\alpha}-n_{\beta}$ as in Eq.(2.13).

In the interface region $|r-R| \lesssim \xi$, the generalized chemical potential $\mu-C \nabla^{2} \phi$ including the gradient term may be treated as a constant $h_{R}$, so that

$$
h_{R}=h-C\left(\frac{\partial^{2}}{\partial r^{2}}+\frac{2}{r} \frac{\partial}{\partial r}\right) \phi .
$$

The solute chemical potential $\mu$ is also a constant $\mu_{R}$ near the interface. We set $\phi=\phi_{\text {int }}(r-R)+\delta \phi$ and $n=n_{\text {int }}(r-R)+\delta n$ in the interface region. Here $\phi_{\text {int }}(z)$ is the one-dimensional interface solution of Eq.(3.4) and $n_{\text {int }}(z)=n_{\beta} e^{g\left(\phi_{\text {int }}(z)-\phi_{\beta}\right)}$. These represent the reference profiles and the corresponding $h$ and $\mu$ are written as $h_{\mathrm{cx}}$ and $\mu_{\mathrm{cx}}$ as in Eq.(5.14). To linear order in $\delta \phi$ and $\delta n$, we obtain $\mu_{R}-\mu_{\mathrm{cx}}=T \delta n / n_{\text {int }}-T g \delta \phi$ or

$$
\delta n=n_{\mathrm{int}}\left[\left(\mu_{R}-\mu_{\mathrm{cx}}\right) / T+g \delta \phi\right],
$$


in the region $|r-R| \lesssim \xi$. We may linearize Eq.(B5) as

$$
\begin{aligned}
& h_{R}-h_{\mathrm{cx}}=\left[f^{\prime \prime}\left(\phi_{\mathrm{int}}\right)-T g^{2} n_{\mathrm{int}}-C \frac{\partial^{2}}{\partial r^{2}}\right] \delta \phi \\
& -\frac{2}{R} C \phi_{\mathrm{int}}^{\prime}-g\left(\mu_{R}-\mu_{\mathrm{cx}}\right) n_{\mathrm{int}}
\end{aligned}
$$

Then we multiply Eq.(B7) by $\phi_{\text {int }}^{\prime}=d \phi_{\text {int }}(r-R) / d r$ and integrate over the region $|r-R| \lesssim \xi$ to obtain

$$
\left(h_{R}-h_{\mathrm{cx}}\right) \Delta \phi \cong \frac{2 \sigma}{R}-\left(\mu_{R}-\mu_{\mathrm{cx}}\right) \Delta n
$$

where the first term in the right hand side of Eq.(B7) does not contribute and use has been made of Eq.(3.9) and $g n_{\text {int }} \phi_{\text {int }}^{\prime}=d n_{\text {int }} / d r$. We rewrite Eq.(B8) as

$$
\left(h_{R}-\bar{h}\right) \Delta \phi+\left(\mu_{R}-\bar{\mu}\right) \Delta n \cong \frac{2 \sigma}{R}-w,
$$

where $w$ is given in Eq.(5.16) in the linear form. Using Eq.(B4) we eliminate $h_{R}-\bar{h}$ and $\mu_{R}-\bar{\mu}$ from Eq.(B9) to obtain the droplet evolution equation (5.19) with

$$
1 / \Lambda=(\Delta \phi)^{2} / L+(\Delta n)^{2} / D_{s} \bar{n} .
$$

where the second term in the right hand side is negligible for dilute solute, leading to $\Lambda \cong L /(\Delta \phi)^{2}$ and Eq.(5.20).
[1] E.L. Eckfeldt and W.W. Lucasse, J. Phys. Chem. 47, 164 (1943); B.J. Hales, G.L. Bertrand, and L.G. Hepler, J. Phys. Chem. 70, 3970 (1966).

[2] V. Balevicius and H. Fuess, Phys. Chem. Chem. Phys. 1 ,1507 (1999).

[3] T. Narayanan and A. Kumar, Phys. Rep. 249, 135 (1994).

[4] J. Jacob, A. Kumar, S. Asokan, D. Sen, R. Chitra, and S. Mazumder, Chem. Phys. Lett. 304, 180 (1999).

[5] M. Misawa, K. Yoshida, K. Maruyama, H. Munemura, and Y. Hosokawa, J. of Phys. and Chem. of Solids 60, 1301(1999).

[6] J. Jacob, A Kumar, M.A. Anisimov, A.A. Povodyrev and J.V. Sengers, Phys. Rev. E 58, 2188 (1998); M. A. Anisimov, J. Jacob, A. Kumar, V. A. Agayan, and J. V. Sengers, Phys. Rev. Lett. 85, 2336 (2000).

[7] T. Takamuku, A. Yamaguchi, D. Matsuo, M. Tabata, M. Kumamoto,J. Nishimoto, K. Yoshida, T. Yamaguchi, M. Nagao, T. Otomo, and T. Adachi, J. Phys. Chem. B 105, 6236 (2001).

[8] K. Jurkiewicz, Fluid Phase Equilibria 251, 24 (2007).

[9] G. W. Euliss and C. M. Sorensen, J. Chem. Phys. 80, 4767 (1984); A. F. Kostko, M. A. Anisimov, and J. V. Sengers, Phys. Rev. E 70, 026118 (2004); M. Wagner, O. Stanga, and W. Schröer, Phys. Chem. Chem. Phys. 6, 580 (2004). C. Yang, W. Li, and C. Wu, J. Phys. Chem. B 108, 11866 (2004); M. Sedlak, J. Phys. Chem. B 110, 4329, 4339, 13976 (2006).

[10] J. Jacob, M. A. Anisimov, J. V. Sengers, A. Oleinikova, H. Weingärtner, and A. Kumar, Phys. Chem. Chem. Phys. 3, 829 (2001).

[11] The dynamic scattering intensity $G(q, t)$ consists of the contribution from the composition fluctuations and that from the heterogeneities. The latter is proportional to $\phi_{\mathrm{h}} R^{3} \exp \left(-D_{R} q^{2} t\right)$ for wave number $q$. Here the heterogeneities have a radius $R \ll 2 \pi / q$ at a volume fraction $\phi_{\mathrm{h}} \ll 1$. The diffusion constant $D_{R}$ is given by the Stokes formula $\left(\propto R^{-1}\right)$.

[12] D. Bonn, D. Ross, S. Hachem, S. Gridel, and J. Meunier, Europhys. Lett., 58, 74 (2002).

[13] K. Sadakane, H. Seto, H. Endo, and M. Shibayama, J. Phys. Soc. Jpn., 76, 113602 (2007); K. Sadakane,, A. Onuki, K. Nishida, S. Koizumi, and H. Seto, Phys. Rev. Lett. 103, 167803 (2009).

[14] P. G. Arscott, C. Ma, J. R. Wenner and V. A. Bloom- field, Biopolymers, 36, 345 (1995); A. Hultgren and D. C. Rau, Biochemistry 43, 8272 (2004); C. Stanley and D. C. Rauy, Biophy. J. 91, 912 (2006).

[15] M. E. Leunissen, A. van Blaaderen, A. D. Hollingsworth, M. T. Sullivan, and P. M. Chaikin, Proc. Natl. Acad. Sci. U.S.A. 104, 2585 (2007); M. E. Leunissen, J. Zwanikken, R. van Roij, P. M. Chaikin, and A. van Blaaderen, Phys. Chem. Chem. Phys. 9, 6405 (2007).

[16] J. de Graaf, J. Zwanikken, M. Bier, A. Baarsma, Y. Oloumi, M. Spelt, and R. van Roij, J. Chem. Phys. 129, 194701 (2008).

[17] J. N. Israelachvili, Intermolecular and Surface Forces (Academic Press, London, 1991).

[18] T. Osakai and K. Ebina, J. Phys. Chem. B 102, 5691 (1998).

[19] D. N. Shin, J. W. Wijnen, J. B. F. N. Engberts, and A. Wakisaka, J. Phys. Chem. B 106, 6014 (2002); H. Kobara, A. Wakisaka, K. Takeuchi, and T. Ibusuki, ibid. 107, 11827 (2003).

[20] J.L. Tveekrem and D.T. Jacobs, Phys. Rev. A 27, 2773 (1983).

[21] D. Beaglehole, J. Phys. Chem. 87, 4749 (1983). See Ref. 37] for discussions on this work.

[22] A. Onuki and H. Kitamura, J. Chem. Phys. 121, 3143 (2004).

[23] A. Onuki, Phys. Rev. E 73, 021506 (2006); J. Chem. Phys. 128, 224704 (2008).

[24] G. Marcus, S. Samin, and Y. Tsori, J. Chem. Phys. 129, 061101 (2008).

[25] M. Bier, J. Zwanikken, and R. van Roij, Phys. Rev. Lett. 101, 046104 (2008); J. Zwanikken, J. de Graaf, M. Bier, and R. van Roij, J. Phys.: Condens. Matter 20, 494238 (2008).

[26] D. Ben-Yaakov, D. Andelman, D. Harries, and R. Podgornik, J. Phys. Chem. B 10, 6001(2009).

[27] T. Araki and A. Onuki, J. Phys.: Condens. Matter 21, 424116 (2009); A. Onuki, T. Araki, and R. Okamoto, to be published in J. Phys.: Condens. Matter (2010).

[28] B. Rotenberg, I. Pagonabarragac, and D. Frenkel, Faraday Discuss., 144, 223 (2010).

[29] A. Onuki and R. Okamoto, J. Phys. Chem. B, 113, 3988 (2009); R. Okamoto and A. Onuki, J. Chem. Phys., 131, 094905 (2009).

[30] A. Onuki, Europhys. Lett. 82, 58002 (2008).

[31] A. Onuki, in Polymer, Liquids and Colloids in Electric 
Fields: Interfacial Instabilities, Orientation and PhaseTransitions ed Y Tsori (World Scientific, Singapore, 2009).

[32] A. Onuki, Phase Transition Dynamics (Cambridge University Press, Cambridge, 2002)

[33] L. Q. Hung, J. Electroanal. Chem. 115, 159 (1980).

[34] L. Onsager and N. N. T. Samaras, J. Chem. Phys. 2, 528 (1934); Y. Levin and J. E. Flores-Mena, Europhys. Lett. 56, 187 (2001).

[35] H. Kitamura and A. Onuki, J. Chem. Phys., 123, 124513 (2005).

[36] J. W. Cahn, J. Chem. Phys. 663667 (1977).
[37] P.G. de Gennes, Rev.Mod.Phys. 57, 827 (1985).

[38] J.S. Langer and A.J. Schwartz, Phys. Rev. A21, 948 (1980).

[39] A.J. Schwartz, S. Krishnamurthy and W.I. Goldburg, Phys. Rev. A 21, 1331 (1980); R.G. Howland, N.-C. Wong and C.M. Knobler, J. Chem. Phys. 73, 522 (1980).

[40] E.D. Siebert and C.M. Knobler, Phys. Rev. Lett. 52, 1133 (1984).

[41] S. B. Kiselev and J. F. Ely, J. Chem. Phys. 119, 8645 (2003). 